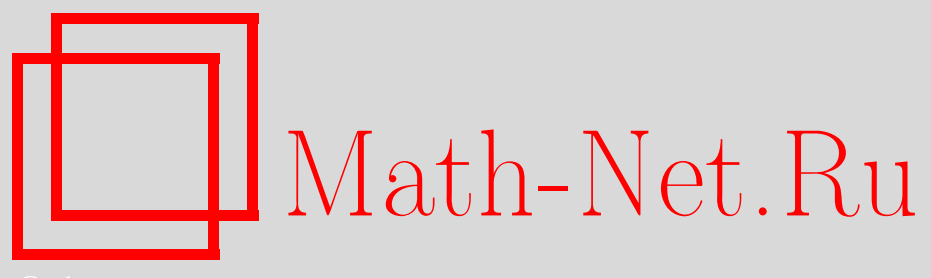

B. А. Мирзоян, Структурные теоремы для Ricполусимметрических подмногообразий и геометрическое описание одного класса минимальных полуэйнштейновых подмногообразий, Матем. сб., 2006, том 197, номер $7,47-76$

DOI: https://doi.org/10.4213/sm1592

Использование Общероссийского математического портала Math-Net.Ru подразумевает, что вы прочитали и согласны с пользовательским соглашением http: //www . mathnet.ru/rus/agreement

Параметры загрузки:

IP: 52.90 .164 .192

26 апреля 2023 г., 15:02:50

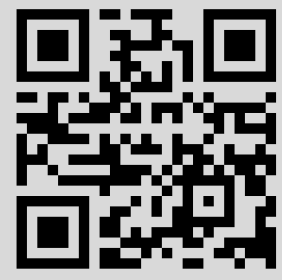




\author{
В. А. Мирзоян
}

\title{
Структурные теоремы для Ric-полусимметрических подмногообразий и геометрическое описание одного класса минимальных полуэйнштейновых подмногообразий
}

\begin{abstract}
В работе доказываются структурные теоремы для подмногообразий с полупараллельным тензором Риччи в евклидовых пространствах при некоторых дополнительных условиях. Более подробно исследуются минимальные подмногообразия. Дается геометрическое описание одного класса нормально плоских минимальных полуэйнштейновых подмногообразий с кратными главными векторами кривизны.
\end{abstract}

Библиография: 36 названий.

\section{§ 0. Введение}

Римановы Ric-полусимметрические пространства характеризуются условием полупараллельности тензора Риччи $R_{1}\left(R(X, Y) R_{1}=0\right)$ и являются естественными обобщениями полусимметрических пространств (характеризуемых условием $R(X, Y) R=0$, где $R$ - тензор кривизны), римановых пространств с параллельным тензором Риччи $\left(\nabla R_{1}=0\right)$ и, в частности, эйнштейновых и симметрических пространств. На протяжении последних сорока лет этот класс пространств был предметом исследований многих авторов [1]-[16]. Для них общая классификационная теорема доказана автором в [8], где были выделены полуэйнштейновы пространства. Примерами полуэйнштейновых пространств служат конусы над эйнштейновыми пространствами (подробности см. в [10], [14]). В статье [12], посвященной классификации Ric-полусимметрических гиперповерхностей в евклидовых пространствах, дано геометрическое описание полуэйнштейновой гиперповерхности, которая представляет собой конус над эйнштейновым пространством. Последнее, в свою очередь, является прямым произведением двух сфер и принадлежит гиперсфере объемлющего пространства. В [14] такой же конус уже выступает в качестве полуэйнштейнова подмногообразия коразмерности $\geqslant 2$. Другие полуэйнштейновы подмногообразия коразмерности $\geqslant 2$, не удовлетворяющие более сильным условиям $\nabla R_{1}=0$ или $R(X, Y) R=0$, пока неизвестны. Вообще, Ric-полусимметрические подмногообразия, за исключением некоторых их частных классов [17]-[19], мало исследованы, и их систематическая теория еще не разработана.

B настоящей работе исследуется проблема приводимости Ric-полусимметрических подмногообразий в евклидовых пространствах и доказывается ряд структурных теорем. Дается геометрическое описание одного класса нормально плоских минимальных полуэйнштейновых подмногообразий $\mathrm{c}$ кратными главными векторами кривизны.

(C) В. А. Мирзоян, 2006 
Геометрия Ric-полусимметрических подмногообразий интересна еще и в том отношении, что эти подмногообразия являются обобщениями подмногообразий с различными параллельными и полупараллельными тензорными полями и представляют собой Ric-огибающие (в точках касания совпадают тензоры Риччи) семейств подмногообразий с параллельным тензором Риччи и, в частности, эйнштейновых подмногообразий и их произведений (см. [9], [11], [13], [20]).

Следует отметить, что в последнее время условия

$$
R(X, Y) R=0, \quad R(X, Y) R_{1}=0
$$

и некоторые их обобщения интенсивно исследуются в полуримановой геометрии и в теории различных классов комплексных и контактных многообразий (см. [21]-[23] и цитированную в них литературу).

\section{§ 1. Основные определения, $V$ - и $Z$-разложения}

Напомним вкратце основные определения и, следуя 3. Сабо [24], опишем так называемые $V$ - и $Z$-разложения касательного пространства риманова многообразия.

Пусть $M$ является $m$-мерным гладким римановым многообразием с римановой метрикой $g$ и римановой связностью $\nabla$. Тензор кривизны $R$ связности $\nabla$ определяется равенством

$$
R(X, Y) Z=\nabla_{X} \nabla_{Y} Z-\nabla_{Y} \nabla_{X} Z-\nabla_{[X, Y]} Z
$$

где $X, Y, Z$ - произвольные касательные к $M$ векторные поля. Выражения $R(X, Y)=\nabla_{X} \nabla_{Y}-\nabla_{Y} \nabla_{X}-\nabla_{[X, Y]}$ называются операторами кривизны связности $\nabla$. Они действуют как дифференцирования тензорной алгебры на $M$. Например, если $Q$ - тензорное поле типа $(1,1)$, то $(R(X, Y) Q) Z=$ $R(X, Y) Q(Z)-Q(R(X, Y) Z)$. Тензор Риччи $R_{1}$ многообразия $M$ определяется стандартным образом: если $\left(e_{1}, \ldots, e_{m}\right)$ - локальный базис ортонормальных касательных векторных полей на $M$, то для любого касательного векторного поля $X$ полагаем

$$
R_{1}(X)=\sum_{i=1}^{m} R\left(X, e_{i}\right) e_{i}
$$

В каждой точке $x \in M$ тензор $R_{1}$ действует как симметрический эндоморфизм касательного пространства $T_{x}(M)$. Если $R_{1}=0$, то риманово многообразие называется риччи-плоским. Если $R_{1}=\lambda I$, где $\lambda$ = const, $I$ - тождественное преобразование, то многообразие $M$ называется эйнштейновым. Теории эйнштейновых многообразий посвящены монографии [25], [26].

Тензорное поле $Q$, определенное на римановом многообразии $M$, называется параллельным, если $\nabla Q=0$ или, более подробно, $\nabla_{X} Q=0$ для любого $X$. Если же $R(X, Y) Q=0$ для любых $X, Y$, то тензорное поле $Q$ называется полупараллельным.

Римановы многообразия с параллельным тензором кривизны называются локально симметрическими, а с полупараллельным тензором кривизны - полусимметрическими. Римановы многообразия с полупараллельным тензором 
Риччи называются Ric-полусимметрическими. В силу импликаций

$$
\begin{array}{rlrll}
\nabla R=0 \quad & \Rightarrow & \nabla R_{1}=0 & \Rightarrow & R(X, Y) R_{1}=0 \\
\nabla R=0 & \Rightarrow & R(X, Y) R=0 & \Rightarrow & R(X, Y) R_{1}=0
\end{array}
$$

класс Ric-полусимметрических многообразий содержит в качестве подклассов локально симметрические и полусимметрические многообразия, многообразия с параллельным тензором Риччи и, в частности, эйнштейновы многообразия.

Общая классификационная теорема римановых Ric-полусимметрических многообразий гласит, что гладкое риманово многообразие $M$ удовлетворяет условию $R(X, Y) R_{1}=0$ тогда и только тогда, когда оно является либо двумерным, либо эйнштейновым, либо полуэйнштейновым, либо прямым произведением (локально) перечисленных классов многообразий [8].

Полуэйнштейновы многообразия определяются следующим образом. Пусть $M$ - риманово многообразие, а $x \in M-$ произвольная точка. Подпространство $T_{x}^{(0)}$ касательного пространства $T_{x}(M)$, определенное равенством $T_{x}^{(0)}=$ $\left\{X \in T_{x}(M): R(X, Y)=0 \forall Y \in T_{x}(M)\right\}$, называется пространством дефектности в точке $x$, а его размерность $\mu_{x}=\operatorname{dim} T_{x}^{(0)}-$ индексом дефектности в этой точке. Пространство $T_{x}^{(0)}$ было определено С. Чженем и Н. Кейпером [27]. Они доказали, что распределение $T^{(0)}$ (распределение дефектности) инволютивно и вполне геодезично, а его интегральное многообразие является локально евклидовым в индуцированной метрике.

Пространство $T_{x}^{(0)}$ всегда содержится в подпространстве собственных векторов тензора $R_{1}$, отвечающих нулевому собственному значению. Ортогональное дополнение $T_{x}^{(1)}$ пространства $T_{x}^{(0)}$ в $T_{x}(M)$ относительно метрики $g$ называется пространством кодефектности многообразия $M$ в точке $x$, а его размерность индексом кодефектности (или просто кодефектностью) в этой точке. Пространство $T_{x}^{(1)}$ инвариантно относительно операторов $R(X, Y)$ и тензора $R_{1}$. Следовательно, в каждой точке $x \in M$ тензор Риччи $R_{1}$ имеет два инвариантных подпространства $T_{x}^{(0)}$ и $T_{x}^{(1)}$ и справедливо разложение в прямую сумму $T_{x}(M)=T_{x}^{(0)}+T_{x}^{(1)}$ (подробности см. в [8]).

Риманово многообразие $M$ с ненулевым индексом дефектности в каждой точке $x$ называется полуэйнштейновым, если его тензор Риччи $R_{1}$ на каждом инвариантном подпространстве $T_{x}^{(1)}$ имеет только одно ненулевое собственное значение.

Примерами полуэйнштейновых многообразий являются римановы многообразия кодефектности два, которые исследовались в [28], а также, как это указывалось выше, конусы над эйнштейновыми многообразиями.

В 1925 г. П. А. Широковым [29] было доказано, что риманово многообразие с параллельным тензором Риччи локально является прямым произведением эйнштейновых многообразий. Эта структурная теорема была распространена автором [30] на нормально плоские подмногообразия с параллельным тензором Риччи в евклидовых пространствах. В связи с тем, что в теории Ric-полусимметрических подмногообразий вопросы приводимости также играют важную роль, в 22 мы докажем ряд структурных теорем для этого класса подмногообразий в объемлющем евклидовом пространстве при некоторых дополнительных условиях. Доказательство этих теорем основано на $V$ - и $Z$-разложениях 
касательного пространства риманова многообразия. В связи с этим здесь мы напомним конструкцию этих разложений и опишем некоторые их свойства.

Пусть $M$ - риманово многообразие, и пусть $x \in M$ - фиксированная точка. В линейном пространстве кососимметрических линейных операторов

$$
T_{x}(M) \rightarrow T_{x}(M)
$$

рассмотрим линейное подпространство $h_{x}$, натянутое на элементы $R_{x}(X, Y)$, где $X, Y \in T_{x}(M)$, т.е. $h_{x}=\operatorname{span} R_{x}(X, Y)$. Для произвольных двух элементов $R_{x}(X, Y)$ и $R_{x}(Z, W)$ из $h_{x}$ определим коммутатор по формуле

$$
\left[R_{x}(X, Y), R_{x}(Z, W)\right]=R_{x}(X, Y) \cdot R_{x}(Z, W)-R_{x}(Z, W) \cdot R_{x}(X, Y) .
$$

Обозначим через $\bar{h}_{x}$ алгебру Ли, порожденную множеством $h_{x}$ относительно этой операции, и пусть $P_{x}-$ связная подгруппа группы изометрий в $T_{x}(M)$, определенная алгеброй Ли $\bar{h}_{x}$. Эта группа называется примитивной группой голономии в точке $x$. Пусть

$$
T_{x}(M)=V_{x}^{(0)}+V_{x}^{(1)}+\cdots+V_{x}^{(t)}
$$

является неприводимым разложением пространства $T_{x}(M)$ относительно $P_{x}$. Подпространства $V_{x}^{(\rho)}$ инвариантны относительно действия $P_{x}$ и попарно вполне ортогональны. Более того, $P_{x}$ действует на $V_{x}^{(0)}$ тривиально, а на $V_{x}^{(\rho)}$, $\rho>0$, неприводимо.

Разложение (1.1) называется $V$-разложением пространства $T_{x}(M)$. Легко видеть, что в этом разложении $V_{x}^{(0)}$ есть не что иное, как пространство дефектности $T_{x}^{(0)}$ многообразия $M$ в точке $x$, определенное выше.

Tеорема 1.1 (3. Сабо [24]). На римановом многообразии $M$ класса $C^{\infty}$ существует всюду плотное открытое множество $G$, на котором размерности подпространств $V_{x}^{(\rho)}$ постоянны, разложение (1.1) единственно с точностъю до порядка прямых слагаемых, а соответствующие распределения $V^{(\rho)}$ обладают на $G$ следующими свойствами:

$$
\begin{aligned}
& \nabla_{V^{(0)}} V^{(0)} \subseteq V^{(0)}, \quad \nabla_{V^{(0)}} V^{(\rho)} \subseteq V^{(\rho)}, \\
& \nabla_{V^{(\rho)}} V^{(0)} \subseteq V^{(0)}+V^{(\rho)}, \quad \nabla_{V^{(\rho)}} V^{(\rho)} \subseteq V^{(0)}+V^{(\rho)}, \\
& \nabla_{V^{(\rho)}} V^{(\tau)} \subseteq V^{(\tau)}, \quad \rho \neq \tau, \quad \rho, \tau \neq 0,
\end{aligned}
$$

где записъ $\nabla_{V^{(\rho)}} V^{(\tau)} \subseteq V^{(\sigma)}$ обозначает, что для любого $X \in V^{(\rho)}$ и любого $Y \in V^{(\tau)}$ вектор $\left(\nabla_{X} Y\right)_{x}$ принадлежст $V_{x}^{(\sigma)}$.

Из приведенных в этой теореме включений следует, что распределения $V^{(\rho)}$, вообще говоря, не являются параллельными на $M$. Однако их всегда можно расширить до параллельных распределений методом 3. Сабо [24], суть которого состоит в следующем. Пусть $Z_{x}^{(\rho)}$ - подпространство в $T_{x}(M)$, натянутое на векторы

$$
\left.X_{1}\right|_{x},\left.\nabla_{X_{1}} X_{2}\right|_{x},\left.\nabla_{X_{1}} \nabla_{X_{2}} X_{3}\right|_{x}, \ldots,\left.\nabla_{X_{1}} \ldots \nabla_{X_{k}} X_{k+1}\right|_{x}, \ldots
$$


где все $X_{k}$ принадлежат $V^{(\rho)}, \rho>0$. По определению полагаем

$$
Z_{x}^{(0)}=\left(Z_{x}^{(1)}+\cdots+Z_{x}^{(t)}\right)^{\perp}
$$

где $(\cdot)^{\perp}$ обозначает ортогональное дополнение в $T_{x}(M)$. Легко видеть, что $Z_{x}^{(0)} \subseteq V_{x}^{(0)}, V_{x}^{(\rho)} \subseteq Z_{x}^{(\rho)}, \rho>0$. Из включений, фигурирующих в теореме 1.1 , следует, что расширение подпространства $V_{x}^{(\rho)}$ до $Z_{x}^{(\rho)}$ происходит только за счет подпространства $V_{x}^{(0)}$.

Теорема 1.2 (З. Сабо [24]). Подпространства $Z_{x}^{(\rho)}, x \in G$, попарно вполне ортогональны, и существует всюду плотное открытое множество $\bar{G} \subset$ $G \subset M$, на котором $Z_{x}^{(\rho)}$ имеют постоянные размерности, а соответствующие распределения $Z^{(\rho)}$ параллельны в римановой связности на $M$.

Разложение $T_{x}(M)=Z_{x}^{(0)}+Z_{x}^{(1)}+\cdots+Z_{x}^{(t)}$ называется $Z$-разложением пространства $T_{x}(M)$.

\section{§ 2. Структурные теоремы для Ric-полусимметрических подмногообразий}

Пусть $M$ является $m$-мерным подмногообразием $n$-мерного евклидова пространства $E_{n}$, и пусть $\langle\cdot, \cdot\rangle$ обозначает скалярное произведение в $E_{n}$, а $g-$ индуцированную метрику на $M$. Если $\widetilde{\nabla}$ и $\nabla$ - римановы связности на $E_{n}$ и $M$, то для любых касательных к $M$ векторных полей $X, Y$ и любого нормального к $M$ векторного поля $\xi$ полагаем

$$
\widetilde{\nabla}_{X} Y=\nabla_{X} Y+\alpha_{2}(X, Y), \quad \widetilde{\nabla}_{X} \xi=-A_{\xi}(X)+\nabla_{X}^{\perp} \xi
$$

где в правых частях $\nabla_{X} Y$ и $A_{\xi}(X)$ обозначают касательную, а $\alpha_{2}(X, Y)$ и $\nabla_{X}^{\perp} \xi-$ нормальную компоненту ковариантных производных, стоящих в левых частях. Эти формулы называются формулами Гаусса и Вейнгартена соответственно. Связность $\nabla$ является связностью Леви-Чивита индуцированной на $M$ метрики $g$, а $\alpha_{2}$ - билинейной симметрической формой, определенной на $T(M) \times T(M)(T(M)$ - касательное расслоение) со значениями в нормальном расслоении $T^{\perp}(M)$. Форма $\alpha_{2}$ называется второй фундаментальной формой подмногообразия $M$. Выражение $A_{\xi}(X)$ линейно по каждому аргументу и связано с $\alpha_{2}$ по формуле $g\left(A_{\xi}(X), Y\right)=\left\langle\alpha_{2}(X, Y), \xi\right\rangle$. Отсюда следует, что в каждой точке $x \in M$ для любого $\xi \in T_{x}^{\perp}(M) A_{\xi}$ является симметрическим эндоморфизмом касательного пространства $T_{x}(M)$. Он называется вторым фундаментальным тензором, соответствующим нормальному векторному полю $\xi$. В формуле Вейнгартена нормальная компонента $\nabla_{X}^{\perp} \xi$ от $\widetilde{\nabla}_{X} \xi$ определяет в $T^{\perp}(M)$ некоторую метрическую связность $\nabla^{\perp}$, называемую нормальной связностью. Тензоры кривизны $R$ и $R^{\perp}$ связностей $\nabla, \nabla^{\perp}$ определяются равенствами

$$
\begin{aligned}
R(X, Y) Z & =\nabla_{X} \nabla_{Y} Z-\nabla_{Y} \nabla_{X} Z-\nabla_{[X, Y]} Z \\
R^{\perp}(X, Y) \xi & =\nabla_{X}^{\perp} \nabla_{Y}^{\perp} \xi-\nabla_{Y}^{\perp} \nabla_{X}^{\perp} \xi-\nabla_{[X, Y]}^{\perp} \xi
\end{aligned}
$$

Если $R=0$, то подмногообразие называется локально евклидовым. Если $R^{\perp}=0$, то говорят о подмногообразии с плоской нормальной связностью или 
просто о нормально плоском подмногообразии. Из уравнения Риччи

$$
\left\langle R^{\perp}(X, Y) \xi, \eta\right\rangle=g\left(\left[A_{\xi}, A_{\eta}\right] X, Y\right)
$$

следует, что $R^{\perp}=0$ тогда и только тогда, когда $\left[A_{\xi}, A_{\eta}\right]=A_{\xi} A_{\eta}-A_{\eta} A_{\xi}=0$ для любых нормальных векторных полей $\xi, \eta$, что равносильно также одновременной диагонализуемости всех тензоров $A_{\xi}$.

Тензор Риччи $R_{1}$ подмногообразия $M$ определяется через тензор кривизны $R$ так же, как и для риманова многообразия.

Если $\alpha_{2}=0$, то подмногообразие $M$ называется вполне геодезическим. В $E_{n}$ вполне геодезическое подмногообразие является плоскостью соответствующей размерности. Если $A_{\xi}=\lambda I$, где $\lambda$ - некоторая функция, а $I$ - тождественное преобразование, то подмногообразие $M$ называется омбилическим относительно нормального векторного поля $\xi$. Вектор средней кривизны $H$ определяется равенством $H=\operatorname{tr} \alpha_{2}$. Если $H=0$, то подмногообразие называется минимальным. Омбилическое относительно $H$ подмногообразие называется псевдоомбилическим. Омбилическое относительно любого нормального векторного поля подмногообразие называется вполне омбилическим. В $E_{n}$ вполне омбилическое подмногообразие является сферой или плоскостью (если $A_{\xi}=0 \forall \xi$ ) соответствующей размерности.

Остальные сведения по геометрии подмногообразий можно найти в [31]-[34].

Изометрическое погружение $M \rightarrow E_{n}$ называется произведением погружений $M_{\varphi} \rightarrow E_{n_{\varphi}}$, если $M=M_{1} \times \cdots \times M_{r}, E_{n}=E_{n_{1}} \times \cdots \times E_{n_{r}}$ и любые два подпространства $E_{n_{\varphi}}$ и $E_{n_{\psi}}, \varphi \neq \psi$, вполне ортогональны в $E_{n}$. В этом случае говорят, что $M$ является прямым произведением подмногообразий $M_{1}, \ldots, M_{r}$ или просто является приводимым.

В вопросах приводимости будем опираться на следующую теорему.

ТеОрема 2.1. Пусть в некоторой области $U$ на подмногообразии $M$ в $E_{n}$ заданы попарно вполне ортогональные инволютивные распределения $\Delta_{1}, \ldots, \Delta_{r}$ $\left(\Delta_{1}(x)+\cdots+\Delta_{r}(x)=T_{x}(U) \quad \forall x \in U\right)$ с интегральными многообразиями $M_{1}, \ldots, M_{r}$ соответственно. Для того чтобы область $U$ была произведением подмногообразий $M_{1}, \ldots, M_{r}$, необходимо и достаточно, чтобъ $\Delta_{1}, \ldots, \Delta_{r}$ были параллельны в связности $\nabla$ на $M$ и сопряжены относительно второй фундаментальной формы $\alpha_{2}\left(\alpha_{2}(X, Y)=0 \forall X \in \Delta_{\varphi}, \forall Y \in \Delta_{\psi}, \varphi \neq \psi\right)$.

О необходимости условий теоремы 2.1 см. [19], достаточность этих условий является содержанием основной леммы Дж. Мура [35].

Перейдем к основной цели настоящего параграфа и докажем ряд структурных теорем для некоторых классов Ric-полусимметрических подмногообразий.

Теорема 2.2. Пусть $M$ является Ric-полусимметрическим подмногообразием с нулевым индексом дефектности в евклидовом пространстве $E_{n}$. Если подпространства собственных векторов (или просто собственные подпространства) тензора Риччи $R_{1}$ в каждой точке сопряжены относительно второй фундаментальной формы $\alpha_{2}$, то $M$ локалъно разлагается в произведение неплоских двумерных подмногообразий и эйнштейновых подмногообразий с нулевым индексом дефектности. 
ДокАЗАТЕЛьСтво. Пусть $\Delta_{1}(x), \ldots, \Delta_{r}(x)$ являются подпространствами собственных векторов (собственными подпространствами) тензора $R_{1}$ в касательном пространстве $T_{x}(M)$, а $\Delta_{1}, \ldots, \Delta_{r}$ обозначают соответствующие распределения (собственные распределения). Известно [8], что $R(X, Y) Z \in \Delta_{\varphi}(x)$ для любого $Z \in \Delta_{\varphi}(x)$ и любых $X, Y, R(X, Y)=0$ для любого $X \in \Delta_{\varphi}(x)$ и любого $Y \in \Delta_{\psi}(x), \varphi \neq \psi, R(X, Y) Z=0$ для любых $X, Y \in \Delta_{\varphi}(x)$ и любого $Z \in \Delta_{\psi}(x), \varphi \neq \psi$. Последнее означает, что эндоморфизмы $R(X, Y)$, $X, Y \in \Delta_{\varphi}(x)$, действуют на $\Delta_{\psi}(x), \psi \neq \varphi$, тривиальным образом.

Точно так же, как и в $\S 1$, получается линейное подпространство $h_{x}^{(\varphi)}=$ $\operatorname{span} R(X, Y), X, Y \in \Delta_{\varphi}(x)$, в линейном пространстве всех кососимметрических эндоморфизмов $\Delta_{\varphi}(x) \rightarrow \Delta_{\varphi}(x)$. Подпространство $h_{x}^{(\varphi)}$ превращается в алгебру Ли относительно скобочной операции, определенной в $\S 1$, и является подалгеброй алгебры $h_{x}$. Следовательно, примитивная группа голономии $P_{x}$ приводима и имеет место разложение

$$
P_{x}=P_{x}^{(1)} \times P_{x}^{(2)} \times \cdots \times P_{x}^{(r)},
$$

где $P_{x}^{(\varphi)}$ - подгруппа с алгеброй Ли $h_{x}^{(\varphi)}$. Подгруппа $P_{x}^{(\varphi)}$ действует на $\Delta_{\psi}(x)$, $\psi \neq \varphi$, тривиально, а на $\Delta_{\varphi}(x)$ она, вообще говоря, может быть приводимой. Пусть $\Delta_{1}(x)$ является собственным подпространством тензора $R_{1}$, отвечающим нулевому собственному значению, и пусть

$\Delta_{1}(x)=V_{x}^{(0)}+V_{x}^{(1,1)}+\cdots+V_{x}^{\left(1, s_{1}\right)}, \quad \Delta_{\varphi}(x)=V_{x}^{(\varphi, 1)}+\cdots+V_{x}^{\left(\varphi, s_{\varphi}\right)}, \quad \varphi>1$, являются неприводимыми разложениями $\Delta_{1}(x)$ и $\Delta_{\varphi}(x), \varphi>1$, относительно подгрупп $P_{x}^{(1)}$ и $P_{x}^{(\varphi)}, \varphi>1$, соответственно. Первое разложение написано на основании того, что $V_{x}^{(0)} \subset \Delta_{1}(x)($ см. $\S 1)$. Подпространства $V_{x}^{\left(\varphi, l_{\varphi}\right)}$, $l_{\varphi}=1, \ldots, s_{\varphi}$, инвариантны относительно действия $P_{x}^{(\varphi)}$ (а следовательно, и $P_{x}$ ) и попарно вполне ортогональны. Более того, $P_{x}^{(\varphi)}$ (а следовательно, и $P_{x}$ ) действует на $V_{x}^{(0)}$ тривиально, а на $V_{x}^{\left(\varphi, l_{\varphi}\right)}$ неприводимо. Следовательно, разложение

$$
T_{x}(M)=V_{x}^{(0)}+V_{x}^{(1,1)}+\cdots+V_{x}^{(1, s 1)}+\cdots+V_{x}^{(r, 1)}+\cdots+V_{x}^{\left(r, s_{r}\right)}
$$

является $V$-разложением пространства $T_{x}(M)$. Отметим, что $\operatorname{dim} \Delta_{\varphi}(x) \geqslant 2$ при $\varphi \geqslant 2$, так как если $\operatorname{dim} \Delta_{\varphi}(x)=1$ для некоторого $\varphi$, то $\Delta_{\varphi}(x) \subset V_{x}^{(0)}$.

По условию теоремы $V_{x}^{(0)}$ является нулевым пространством. В этом случае система включений в теореме 1.1 сводится к следующей системе:

$$
\nabla_{V^{(\rho)}} V^{(\rho)} \subseteq V^{(\rho)}, \quad \nabla_{V^{(\rho)}} V^{(\tau)} \subseteq V^{(\tau)}, \quad \rho \neq \tau .
$$

Отсюда следует, что распределения $V^{(\rho)}$ параллельны. На основании этого заключаем, что распределения $V^{\left(\varphi, l_{\varphi}\right)}$ параллельны. Тогда каждое распределение $\Delta_{\varphi}$, являясь суммой таких распределений, также параллельно. Так как $\Delta_{\varphi}(x)$ сопряжены относительно второй фундаментальной формы $\alpha_{2}$ по условию, то на основании теоремы 2.1 заключаем, что подмногообразие $M$ является прямым произведением интегральных многообразий распределений $\Delta_{\varphi}$. Поскольку на каждом подпространстве $\Delta_{\varphi}(x)$ тензор $R_{1}$ имеет только одно собственное значение, то интегральное многообразие распределения $\Delta_{\varphi}$ либо двумерно, либо эйнштейново, с нулевым индексом дефектности в каждом случае. 
Теорема 2.3. Пусть $M$ является нормально плоским Ric-полусимметрическим подмногообразием с нулевым индексом дефектности в евклидовом пространстве $E_{n}$. Тогда $M$ локально разлагается в прямое произведение нормально плоских неплоских двумерных подмногообразий и нормалъно плоских эйнштейновых подмногообразий с нулевым индексом дефектности.

ДокАЗАТЕЛЬСтво. Достаточно заметить, что в случае плоской нормальной связности тензор Риччи $R_{1}$ и все тензоры $A_{\xi}$ одновременно диагонализуются (см. [34], это фактически доказано и в 33 ) и, следовательно, собственные распределения $\Delta_{1}, \ldots, \Delta_{r}$ тензора $R_{1}$ будут сопряжены относительно второй фундаментальной формы $\alpha_{2}$. Теперь утверждение теоремы следует из теоремы 2.2 и того факта, что прямое произведение подмногообразий является нормально плоским тогда и только тогда, когда каждое подмногообразие-сомножитель является нормально плоским [6], [19].

TЕОРема 2.4. Пусть $M$ является нормально плоским Ric-полусимметрическим подмногообразием в $E_{n}$ с ненулевым индексом дефектности. Если собственное распределение тензора Риччи $R_{1}$, отвечающее нулевому собственному значению, является параллельным, то $M$ разлагается в прямое произведение нормально плоских подмногообразий $M^{(1)}, \ldots, M^{(r)}$, где $M^{(1)}$ риччи-плоское с таким же индексом дефектности, что и $M$, а каждое $M^{(\varphi)}$, $\varphi>1$, является либо неплоским двумерным, либо эйнштейновым (но не риччи-плоским) подмногообразием.

ДокАЗАтЕЛьство. Пусть $\Delta_{1}(x)$ - собственное подпространство тензора Риччи $R_{1}$, отвечающее нулевому собственному значению. Так как распределение $\Delta_{1}$ является параллельным на $M$, то его ортогональное дополнение $\Delta_{2}+\cdots+\Delta_{r}$ также будет параллельным. В силу плоской нормальной связности тензор $R_{1}$ коммутирует со всеми тензорами $A_{\xi}$. Поэтому распределения $\Delta_{1}, \Delta_{2}, \ldots, \Delta_{r}$ сопряжены относительно второй фундаментальной формы $\alpha_{2}$ и, следовательно, распределения $\Delta_{1}$ и $\Delta_{2}+\cdots+\Delta_{r}$ также сопряжены относительно $\alpha_{2}$. Отсюда следует, что $M$ является прямым произведением их интегральных многообразий. Интегральное многообразие распределения $\Delta_{1}$ будет риччи-плоским, а для интегрального многообразия распределения $\Delta_{2}+\cdots+\Delta_{r}$ будут выполняться все условия теоремы 2.3.

ТеОрема 2.5. Пусть $M$ является нормально плоским Ric-полусимметрическим подмногообразием в $E_{n}$ с ненулевым индексом дефектности, и пусть распределение дефектности $V^{(0)}$ является омбилическим относительно любого нормалъного векторного поля $\xi$, m.е. $A_{\xi}(X)=\lambda_{\xi} X \forall X \in V_{x}^{(0)}$. Тогда $M$ разлагается в прямое произведение двумерных, эйнштейновых и полуэйнштейновых нормалъно плоских подмногообразий.

ДокАЗАТЕЛЬСтво. Пусть $\Delta_{1}(x), \ldots, \Delta_{r}(x)$ являются пространствами собственных векторов тензора Риччи $R_{1}$. Так как нормальная связность плоская, то $R_{1}$ и все тензоры $A_{\xi}$ одновременно диагонализуются. Это значит, что в подпространствах $\Delta_{1}(x), \ldots, \Delta_{r}(x)$ базисы можно выбрать так, что матрицы всех тензоров $A_{\xi}$ одновременно будут иметь диагональный вид. Будем считать, что базис пространства $T_{x}(M)$ так и выбран и зафиксирован. 
Пусть $\Delta_{1}(x)$ является собственным подпространством, отвечающим нулевому собственному значению тензора $R_{1}$. Так как $V_{x}^{(0)} \subset \Delta_{1}(x)$, то для $\Delta_{1}(x)$ и $\Delta_{\varphi}(x), \varphi>1$, имеют место следующие разложения:

$$
\Delta_{1}(x)=V_{x}^{(0)}+V_{x}^{(1,1)}+\cdots+V_{x}^{\left(1, s_{1}\right)}, \quad \Delta_{\varphi}(x)=V_{x}^{(\varphi, 1)}+\cdots+V_{x}^{\left(\varphi, s_{\varphi}\right)},
$$

которые являются неприводимыми разложениями относительно подгрупп $P_{x}^{(1)}$ и $P_{x}^{(\varphi)}, \varphi>1$, соответственно (см. доказательство теоремы 2.2). Так как на каждом $\Delta_{\varphi}(x)$ все тензоры $A_{\xi}$ имеют диагональный вид, то все $\Delta_{\varphi}(x)$ являются инвариантными относительно тензоров $A_{\xi}$, т.е. если $X \in \Delta_{\varphi}(x)$, то $A_{\xi}(X) \in \Delta_{\varphi}(x)$. Покажем, что $\Delta_{1}(x)$ является прямой суммой двух инвариантных подпространств относительно всех тензоров $A_{\xi}$. Действительно, так как по условию теоремы $V_{x}^{(0)}$ является омбилическим относительно всех тензоров $A_{\xi}$, то оно инвариантно относительно всех $A_{\xi}$. Пусть $X \in V_{x}^{(0)}$, $Y \in V_{x}^{(1,1)}+\cdots+V_{x}^{\left(1, s_{1}\right)}$. Так как подпространства $V_{x}^{(0)}$ и $V_{x}^{\left(\varphi, l_{\varphi}\right)}$ вполне ортогональны (см. [24]), то

$$
g\left(A_{\xi}(Y), X\right)=g\left(Y, A_{\xi}(X)\right)=g\left(Y, \lambda_{\xi} X\right)=0
$$

и, следовательно,

$$
A_{\xi}(Y) \in V_{x}^{(1,1)}+\cdots+V_{x}^{\left(1, s_{1}\right)} .
$$

Таким образом, $V_{x}^{(0)}$ и $V_{x}^{(1,1)}+\cdots+V_{x}^{\left(1, s_{1}\right)}$ являются инвариантными относительно всех тензоров $A_{\xi}$. Это значит, что базисы в $V_{x}^{(0)}$ и $V_{x}^{(1,1)}+\cdots+V_{x}^{\left(1, s_{1}\right)}$ можно выбирать независимо. Поэтому можем считать, что зафиксированный выше базис в $\Delta_{1}(x)$ выбран так, что все $A_{\xi}$ имеют на прямой сумме $V_{x}^{(1,1)}+\cdots+V_{x}^{\left(1, s_{1}\right)}$ диагональный вид. Способом, указанным в $\S 1$, каждое $V_{x}^{\left(\varphi, l_{\varphi}\right)}$ можем расширить до соответствующего параллельного подпространства $Z_{x}^{\left(\varphi, l_{\varphi}\right)}$. Из включений, фигурирующих в теореме 1.1 , следует, что это расширение происходит только за счет подпространства $V_{x}^{(0)}$. Пусть $\widetilde{\Delta}_{\varphi}(x)$ обозначает расширение $\Delta_{\varphi}(x), \varphi>1$. Тогда

$$
\widetilde{\Delta}_{\varphi}(x)=\Delta_{\varphi}(x)+W_{\varphi}(x)
$$

где $W_{\varphi}(x) \subset V_{x}^{(0)}$. Так как на $\Delta_{\varphi}(x), \varphi>1$, все тензоры $A_{\xi}$ имеют диагональный вид, а на $W_{\varphi}(x)$ каждый тензор $A_{\xi}$ имеет только одно собственное значение, то при произвольном выборе базиса в $W_{\varphi}(x)$ все $A_{\xi}$ будут иметь на $\widetilde{\Delta}_{\varphi}(x)$ диагональный вид. В процессе расширения подпространств $\Delta_{\varphi}(x)$, $\varphi>1$, за счет $V_{x}^{(0)}$ подпространство $\Delta_{1}(x)$ может только сузиться до некоторого подпространства $\widetilde{\Delta}_{1}(x)$, которое имеет следующую структуру:

$$
\widetilde{\Delta}_{1}(x)=W_{1}(x)+V_{x}^{(1,1)}+\cdots+V_{x}^{\left(1, s_{1}\right)},
$$

где $W_{1}(x) \subset V_{x}^{(0)}$. Очевидно, что и на $\widetilde{\Delta}_{1}(x)$ все тензоры $A_{\xi}$ имеют диагональный вид. Тогда подпространства $\widetilde{\Delta}_{1}(x), \ldots, \widetilde{\Delta}_{r}(x)$ сопряжены относительно второй фундаментальной формы $\alpha_{2}$. Так как по построению распределения $\widetilde{\Delta}_{1}, \ldots, \widetilde{\Delta}_{r}$ параллельны на подмногообразии $M$, то $M$ разлагается в прямое 
произведение их интегральных многообразий. Интегральное многообразие распределения $\widetilde{\Delta}_{1}$ является риччи-плоским. Если при $\varphi>1 \quad W_{\varphi}(x)$ является нулевым подпространством, то на $\widetilde{\Delta}_{\varphi}(x)$ тензор $R_{1}$ имеет только одно ненулевое собственное значение. Следовательно, интегральное многообразие распределения $\widetilde{\Delta}_{\varphi}$ является либо неплоским двумерным, либо эйнштейновым (но не риччи-плоским) подмногообразием. Если $W_{\varphi}(x)$ не является нулевым подпространством, то тензор $R_{1}$ имеет на $\widetilde{\Delta}_{\varphi}(x), \varphi>1$, только два собственных значения: ненулевое собственное значение на $\Delta_{\varphi}(x)$ и нулевое собственное значение на $W_{\varphi}(x)$. Так как $\Delta_{\varphi}(x)$ при $\varphi>1$ не пересекается с $V_{x}^{(0)}$, то $W_{\varphi}(x)$ фактически является пространством дефектности для интегрального многообразия распределения $\widetilde{\Delta}_{\varphi}(x)$. Следовательно, интегральное многообразие распределения $\widetilde{\Delta}_{\varphi}$ является полуэйнштейновым. Все интегральные многообразия будут иметь плоскую нормальную связность (см. доказательство теоремы 2.3).

На основании теоремы 2.5 можем сформулировать следующую теорему.

Tеорема 2.6. Пусть $M$ является нормально плоским Ric-полусимметрическим подмногообразием в $E_{n}$ c ненулевым индексом дефектности, u пусть собственное распределение $\Delta_{1}$ тензора Риччи $R_{1}$, отвечающее нулевому собственному значению, является омбилическим относительно всех тензоров $A_{\xi}$, m.е. $A_{\xi}(X)=\lambda_{\xi} X \forall X \in \Delta_{1}$. Тогда $M$ разлагается в прямое произведение так же, как и в теореме 2.5.

Для доказательства этой теоремы достаточно заметить, что в каждой точке $x \in M V_{x}^{(0)} \subset \Delta_{1}(x)$ и потому $V_{x}^{(0)}$ является омбилическим относительно всех тензоров $A_{\xi}$. Следовательно, выполняются условия теоремы 2.5 .

Пусть $\alpha_{2}$ является второй фундаментальной формой подмногообразия $M$ в $E_{n}$. Индексом относительной дефектности в точке $x \in M$ называется размерность подпространства $T_{x}^{\prime}$ касательного пространства $T_{x}(M)$, определяемого равенством

$$
T_{x}^{\prime}=\left\{X \in T_{x}(M): \alpha_{2}(X, Y)=0 \forall Y \in T_{x}(M)\right\} .
$$

В силу $\left\langle\alpha_{2}(X, Y), \xi\right\rangle=g\left(A_{\xi}(X), Y\right)$ подпространство $T_{x}^{\prime}$ можем определить следующим эквивалентным способом:

$$
T_{x}^{\prime}=\left\{X \in T_{x}(M): A_{\xi}(X)=0 \forall \xi \in T_{x}^{\perp}(M)\right\} .
$$

Справедливо включение $T_{x} \subset T_{x}^{(0)}[27]$.

Покажем, что распределение $T^{\prime}$ интегрируемо и его интегральное многообразие представляет собой плоскость в $E_{n}$. Действительно, пусть $\bar{\nabla}$ обозначает связность Ван дер Вардена-Бортолотти (см. [34] или §3 настоящей работы). Если $X, Y \in T^{\prime}$, а $Z$ - произвольное касательное векторное поле, то, используя уравнение Кодацци $\left(\bar{\nabla}_{X} \alpha_{2}\right)(Y, Z)=\left(\bar{\nabla}_{Y} \alpha_{2}\right)(X, Z)$, получаем

$$
\begin{aligned}
& \left(\bar{\nabla}_{X} \alpha_{2}\right)(Y, Z)=\nabla_{X}^{\perp} \alpha_{2}(Y, Z)-\alpha_{2}\left(\nabla_{X} Y, Z\right)-\alpha_{2}\left(Y, \nabla_{X} Z\right)=-\alpha_{2}\left(\nabla_{X} Y, Z\right), \\
& \left(\bar{\nabla}_{Y} \alpha_{2}\right)(X, Z)=\nabla_{Y}^{\perp} \alpha_{2}(X, Z)-\alpha_{2}\left(\nabla_{Y} X, Z\right)-\alpha_{2}\left(X, \nabla_{Y} Z\right)=-\alpha_{2}\left(\nabla_{Y} X, Z\right) .
\end{aligned}
$$

Следовательно, $\alpha_{2}\left(\nabla_{X} Y-\nabla_{Y} X, Z\right)=0$, и так как $\nabla_{X} Y-\nabla_{Y} X=[X, Y]$, то $[X, Y] \in T^{\prime}$. 
Это значит, что распределение $T^{\prime}$ интегрируемо. Далее для тех же $X, Y$ и $Z$ имеем

$$
\begin{aligned}
& \left(\bar{\nabla}_{Z} \alpha_{2}\right)(X, Y)=\nabla_{Z}^{\perp} \alpha_{2}(X, Y)-\alpha_{2}\left(\nabla_{Z} X, Y\right)-\alpha_{2}\left(X, \nabla_{Z} Y\right)=0 \\
& \left(\bar{\nabla}_{X} \alpha_{2}\right)(Z, Y)=\nabla_{X}^{\perp} \alpha_{2}\left(\nabla_{X} Z, Y\right)-\alpha_{2}\left(Z, \nabla_{X} Y\right)-\alpha_{2}\left(Z, \nabla_{X} Y\right)=-\alpha_{2}\left(Z, \nabla_{X} Y\right) .
\end{aligned}
$$

Отсюда следует, что $\alpha_{2}\left(Z, \nabla_{X} Y\right)=0$ и в силу произвольности $Z \nabla_{X} Y \in T^{\prime}$. Это значит, что $T^{\prime}$ является геодезическим распределением, т.е. его интегральное многообразие вполне геодезично в $M$. Так как все тензоры $A_{\xi}$ имеют на $T^{\prime}$ только нулевые собственные значения, то интегральное многообразие распределения $T^{\prime}$ является вполне геодезическим в $E_{n}$, т.е. плоскостью.

ТеОРема 2.7. Пусть $M$ является нормально плоским Ric-полусимметрическим подмногообразием в $E_{n}$, и пусть его индекс дефектности совпадает в каждой точке с индексом относительной дефектности. Тогда М разлагается в прямое произведение так же, как и в теореме 2.5.

Доказательство следует из того, что в силу включения $T_{x}^{\prime} \subset T_{x}^{(0)}$ и условия теоремы пространства $T_{x}^{\prime}$ и $T_{x}^{(0)}$ будут совпадать и все тензоры $A_{\xi}$ будут иметь на $T_{x}^{(0)}$ только нулевые собственные значения. Следовательно, выполняются условия теоремы 2.5 .

Структурная теорема для нормально плоских минимальных Ric-полусимметрических подмногообразий сформулирована в 33 .

\section{§ 3. Формализм расслоения адаптированных ортонормреперов подмногообразия и редукция некоторых условий}

Пусть $O\left(E_{n}\right)$ обозначает главное расслоение ортонормированных реперов $\left\{x, e_{1}, \ldots, e_{n}\right\}$ в евклидовом пространстве $E_{n}$. Отождествляя точку $x$ с ее радиус-вектором, будем иметь

$$
d x=\omega^{A} e_{A}, \quad d e_{A}=\omega_{A}^{B} e_{B}, \quad \omega_{B}^{A}+\omega_{A}^{B}=0, \quad A, B, \ldots=1, \ldots, n .
$$

Отсюда путем внешнего дифференцирования получим следующие структурные уравнения:

$$
d \omega^{A}=\omega^{B} \wedge \omega_{B}^{A}, \quad d \omega_{A}^{B}=\omega_{A}^{C} \wedge \omega_{C}^{B}
$$

Пусть $M$ является $m$-мерным подмногообразием в $E_{n}$. Тогда расслоение $O\left(E_{n}\right)$ может быть приведено к главному расслоению $O\left(M, E_{n}\right)$ адаптированных ортонормреперов $\left\{x, e_{1}, \ldots, e_{m}, e_{m+1}, \ldots, e_{n}\right\}$, характеризуемых тем, что

$$
e_{i} \in T_{x}(M), \quad i, j, \ldots=1, \ldots, m, \quad e_{\alpha} \in T_{x}^{\perp}(M), \quad \alpha, \beta, \ldots=m+1, \ldots, n
$$

где $T_{x}(M)$ и $T_{x}^{\perp}(M)$ обозначают соответственно касательное и нормальное к $M$ пространства в точке $x \in M$ (подробности см. в [33]). В силу этого по известной 
схеме [9], [13], [19] получим

$$
\begin{gathered}
\omega^{\alpha}=0, \quad \omega_{i}^{\alpha}=h_{i j}^{\alpha} \omega^{j}, \quad h_{i j}^{\alpha}=h_{j i}^{\alpha}, \\
\bar{\nabla} h_{i j}^{\alpha}=h_{i j k}^{\alpha} \omega^{k}, \quad h_{i j k}^{\alpha}=h_{i k j}^{\alpha}\left(=\bar{\nabla}_{k} h_{i j}^{\alpha}\right), \\
h_{i j k}^{\alpha} \wedge \omega^{k}=h_{i j}^{\beta} \Omega_{\beta}^{\alpha}-h_{k j}^{\alpha} \Omega_{i}^{k}-h_{i k}^{\alpha} \Omega_{j}^{k}, \\
\bar{\nabla} h_{i j}^{\alpha}=d h_{i j}^{\alpha}+h_{i j}^{\beta} \omega_{\beta}^{\alpha}-h_{k j}^{\alpha} \omega_{i}^{k}-h_{i k}^{\alpha} \omega_{j}^{k}, \\
\bar{\nabla} h_{i j k}^{\alpha}=d h_{i j k}^{\alpha}+h_{i j k}^{\beta} \omega_{\beta}^{\alpha}-h_{l k j}^{\alpha} \omega_{i}^{l}-h_{i l k}^{\alpha} \omega_{j}^{l}-h_{i j l}^{\alpha} \omega_{k}^{l} .
\end{gathered}
$$

Здесь $\bar{\nabla}$ обозначает связность Ван дер Вардена-Бортолотти, т.е. $\bar{\nabla}=\nabla \oplus \nabla^{\perp}$, где $\nabla$ является римановой связностью на $M$, определяемой 1-формами $\omega_{i}^{j}$, a $\nabla^{\perp}-$ нормальной связностью, определяемой 1-формами $\omega_{\beta}^{\alpha}$.

В (3.1) и (3.2) функции $h_{i j}^{\alpha}, h_{i j k}^{\alpha}$ являются компонентами второй $\alpha_{2}$ и третьей $\alpha_{3}$ фундаментальных форм соответственно. Их действие определяется следующим образом: если $X=X^{i} e_{i}, Y=Y^{j} e_{j}, Z=Z^{k} e_{k}$, то

$$
\alpha_{2}(X, Y)=h_{i j}^{\alpha} X^{i} Y^{j} e_{\alpha}, \quad \alpha_{3}(X, Y, Z)=h_{i j k}^{\alpha} X^{i} Y^{j} Z^{k} e_{\alpha}
$$

В (3.3) 2-формы

$$
\begin{aligned}
& \Omega_{i}^{j}=d \omega_{i}^{j}-\omega_{i}^{k} \wedge \omega_{k}^{j}=-\sum_{\alpha} h_{i[k}^{\alpha} h_{l] j}^{\alpha} \omega^{k} \wedge \omega^{l}, \\
& \Omega_{\alpha}^{\beta}=d \omega_{\alpha}^{\beta}-\omega_{\alpha}^{\gamma} \wedge \omega_{\gamma}^{\beta}=-\sum_{i} h_{i[k}^{\alpha} h_{l] i}^{\beta} \omega^{k} \wedge \omega^{l}
\end{aligned}
$$

являются формами кривизны связностей $\nabla$ и $\nabla^{\perp}$ соответственно, а коэффициенты

$$
R_{i k l}^{j}=-\sum_{\alpha} h_{i[k}^{\alpha} h_{l] j}^{\alpha}, \quad R_{\alpha k l}^{\beta}=-\sum_{i} h_{i[k}^{\alpha} h_{l] i}^{\beta}
$$

представляют собой компоненты тензоров кривизны $R$ и $R^{\perp}$ этих связностей.

Компоненты $R_{i k}$ тензора Риччи $R_{1}$ определяются по формуле

$$
R_{i k}=R_{i k l}^{l}=\sum_{\alpha}\left(h_{i l}^{\alpha} h_{k}^{\alpha l}-H^{\alpha} h_{i k}^{\alpha}\right),
$$

где $h_{k}^{\alpha l}=h_{k l}^{\alpha}$, а $H^{\alpha}=\sum_{l} h_{l l}^{\alpha}$ - компоненты вектора средней кривизны $H$.

Пусть $T$ - некоторое тензорное поле на подмногообразии $M$ в $E_{n}$, а $T_{i j}^{\alpha}-$ его компоненты в выбранном выше репере. Такое строение компонент не будет умалять общность рассуждений. Первые и вторые ковариантные производные $T_{i j, k}^{\alpha}, T_{i j, k l}^{\alpha}$ тензора $T_{i j}^{\alpha}$ определяются равенствами

$$
\begin{gathered}
\bar{\nabla} T_{i j}^{\alpha}=d T_{i j}^{\alpha}+T_{i j}^{\beta} \omega_{\beta}^{\alpha}-T_{k j}^{\alpha} \omega_{i}^{k}-T_{i k}^{\alpha} \omega_{j}^{k}=T_{i j, k} \omega^{k}, \\
\bar{\nabla} T_{i j, k}^{\alpha}=d T_{i j, k}^{\alpha}+T_{i j, k}^{\beta} \omega_{\beta}^{\alpha}-T_{l j, k}^{\alpha} \omega_{i}^{l}-T_{i l, k}^{\alpha} \omega_{j}^{l}-T_{i j, l}^{\alpha} \omega_{k}^{l}=T_{i j, k l}^{\alpha} \omega^{l} .
\end{gathered}
$$

Дифференцируя внешним образом первое из этих равенств и учитывая второе, получаем

$$
T_{i j, k l}^{\alpha} \omega^{k} \wedge \omega^{l}=T_{p j}^{\alpha} \Omega_{i}^{p}+T_{i p}^{\alpha} \Omega_{j}^{p}-T_{i j}^{\beta} \Omega_{\beta}^{\alpha} .
$$


Условие $\bar{\nabla} T_{i j}^{\alpha}=0$, что равносильно $T_{i j, k}=0$, является условием параллельности тензорного поля $T$, а условие $T_{i j, k l}^{\alpha}=T_{i j, l k}$ - условием его полупараллельности. Из (3.5) следует что условие полупараллельности тензорного поля $T$ равносильно следующему условию:

$$
T_{p j}^{\alpha} \Omega_{i}^{p}+T_{i p}^{\alpha} \Omega_{j}^{p}-T_{i j}^{\beta} \Omega_{\alpha}^{\beta}=0 .
$$

Условие параллельности $T$ коротко записывается в виде $\nabla T=0$. Так как $T_{i j, k l}^{\alpha}=\bar{\nabla}_{l} \bar{\nabla}_{k} T_{i j}^{\alpha}$, то условие полупараллельности $T$ можем записать следующим образом: $\bar{R}(X, Y) \cdot T=0$, где $X, Y$ - произвольные касательные к $M$ векторные поля, а $\bar{R}(X, Y)$ - соответствующие операторы кривизны связности $\bar{\nabla}$.

На основании вышеизложенного условия полупараллельности второй фундаментальной формы $\alpha_{2}$, тензора кривизны $R$, тензора Риччи $R_{1}$ можем представить в следующем виде:

$$
\begin{gathered}
h_{k j}^{\alpha} \Omega_{i}^{k}+h_{i k}^{\alpha} \Omega_{j}^{k}-h_{i j}^{\beta} \Omega_{\beta}^{\alpha}=0, \\
R_{p j k}^{l} \Omega_{i}^{p}+R_{i p k}^{l} \Omega_{j}^{p}+R_{i j p}^{l} \Omega_{k}^{p}-R_{i j k}^{p} \Omega_{p}^{l}=0, \\
R_{k j} \Omega_{i}^{k}+R_{i k} \Omega_{j}^{k}=0 .
\end{gathered}
$$

Путем прямого вычисления легко убедиться в справедливости импликаций $(3.6) \Rightarrow(3.7) \Rightarrow(3.8)$, т.е.

$$
\bar{R}(X, Y) \cdot \alpha_{2}=0 \quad \Rightarrow \quad \bar{R}(X, Y) \cdot R=0 \quad \Rightarrow \quad \bar{R}(X, Y) \cdot R_{1}=0 .
$$

Так как матрица $\left\|R_{j k}\right\|$ является симметрической, то в некотором ортонормрепере она может быть приведена к диагональному виду $\left\|\rho_{j} \delta_{j k}\right\|$ с вещественными диагональными элементами $\rho_{j}$. Тогда из (3.8) получаем

$$
\left(\rho_{j}-\rho_{k}\right) \Omega_{k}^{j}=0
$$

где нет суммирования по $j$. Это равенство также является условием полупараллельности тензора Риччи.

Пусть подмногообразие $M$ является нормально плоским, т.е. $R_{\alpha i j}^{\beta}=0$ или $\Omega_{\beta}^{\alpha}=0$. Тогда

$$
\sum_{k} h_{i k}^{\alpha} h_{k j}^{\beta}=\sum_{k} h_{i k}^{\beta} h_{k j}^{\alpha}
$$

и, следовательно, все матрицы $\left\|h_{i j}^{\alpha}\right\|$ коммутируют между собой. В силу этого в некотором ортонормрепере они одновременно могут быть приведены к диагональному виду $\left\|\lambda_{i}^{\alpha} \delta_{i j}\right\|$. Тогда из (3.6) и (3.4) получаем

$$
\begin{gathered}
\left(\lambda_{i}^{\alpha}-\lambda_{j}^{\alpha}\right) \Omega_{j}^{i}=0, \\
R_{i k}=\sum_{\alpha}\left[\left(\lambda_{i}^{\alpha}\right)^{2}-H^{\alpha} \lambda_{i}^{\alpha}\right] \delta_{i k},
\end{gathered}
$$

где в (3.10) нет суммирования по $i$. Из (3.11) следует, что матрица $\left\|R_{i k}\right\|$ имеет диагональный вид с диагональными элементами

$$
\rho_{i}=\sum_{\alpha}\left[\left(\lambda_{i}^{\alpha}\right)^{2}-H^{\alpha} \lambda_{i}^{\alpha}\right] .
$$


Так как $\Omega_{k}^{j}=-\sum_{\alpha} \lambda_{j}^{\alpha} \lambda_{k}^{\alpha} \omega^{k} \wedge \omega^{j}$, то условия (3.9) и (3.10) окончательно принимают следующий вид:

$$
\begin{aligned}
\left(\rho_{j}-\rho_{k}\right) \sum_{\alpha} \lambda_{j}^{\alpha} \lambda_{k}^{\alpha} & =0, \\
\left(\lambda_{j}^{\alpha}-\lambda_{k}^{\alpha}\right) \sum_{\beta} \lambda_{j}^{\beta} \lambda_{k}^{\beta} & =0,
\end{aligned}
$$

где второй сомножитель в левых частях есть секционная кривизна $k\left(e_{j} \wedge e_{k}\right)$.

Таким образом, в случае плоской нормальной связности необходимые и достаточные условия полупараллельности тензора Риччи и второй фундаментальной формы подмногообразия приводятся соответственно к виду (3.13) и (3.14).

Нормальные векторы $n_{i}=\lambda_{i}^{\alpha} e_{\alpha}$ называются главными векторами кривизны нормально плоского подмногообразия в $E_{n}$. Легко видеть, что $H=n_{1}+\cdots+n_{m}$.

Пусть $\langle\cdot, \cdot\rangle$ - скалярное произведение в $E_{n}$. Тогда

$$
\begin{gathered}
\sum_{\alpha}\left(\lambda_{j}^{\alpha}\right)^{2}=\left\langle n_{j}, n_{j}\right\rangle=\left|n_{j}\right|^{2}, \quad \sum_{\alpha} H^{\alpha} \lambda_{j}^{\alpha}=\left\langle H, n_{j}\right\rangle, \quad \sum_{\alpha} \lambda_{j}^{\alpha} \lambda_{k}^{\alpha}=\left\langle n_{j}, n_{k}\right\rangle, \\
\rho_{j}=\left|n_{j}\right|^{2}-\left\langle H, n_{j}\right\rangle,
\end{gathered}
$$

где $\left|n_{j}\right|$ - модуль вектора $n_{j}$.

Пусть подмногообразие $M$ является минимальным, т.е. $H=0$. Тогда $\rho_{j}=$ $\left|n_{j}\right|^{2}$ и если, например, $n_{1}, \ldots, n_{\nu}=0, n_{\nu+1} \neq 0, \ldots, n_{m} \neq 0$, то индекс относительной дефектности равен $\nu$, т.е. $\operatorname{dim} T_{x}^{\prime}=\nu$. Тогда $\rho_{\nu+1} \neq 0, \ldots, \rho_{m} \neq 0$ и индекс дефектности подмногообразия $M$ также равен $\nu$, т.е. $\mu=\nu$. В силу включения $T_{x}^{\prime} \subset T_{x}^{(0)}$ пространства $T_{x}^{\prime}$ и $T_{x}^{(0)}$ совпадают.

Таким образом, справедлива следующая

ЛЕмма 3.1. Индекс дефектности нормально плоского минималъного подмногообразия $M$ в евклидовом пространстве $E_{n}$ в каждой точке равен индексу относительной дефектности.

На основании леммы 3.1 и теорем 2.7 и 2.5 можем сформулировать следующую теорему.

ТЕОРема 3.1. Пусть $M$ является нормально плоским минимальным Riсполусимметрическим подмногообразием в евклидовом пространстве $E_{n}$. Тогда $M$ разлагается в прямое произведение нормально плоских минимальных подмногообразий $M^{(1)}, \ldots, M^{(r)}$, где каждое $M^{(\varphi)}$ является либо двумерным, либо эйнштейновым, либо полуэйнштейновым.

В этой теореме минимальность каждого $M^{(\varphi)}$ следует из того, что прямое произведение подмногообразий является минимальным тогда и только тогда, когда каждое подмногообразие-сомножитель является минимальным (см., например, [6]).

Условие (3.13) можем представить в следующих двух эквивалентных форmax:

$$
\begin{gathered}
\left(\left|n_{j}\right|^{2}-\left|n_{k}\right|^{2}+\left\langle H, n_{k}-n_{j}\right\rangle\right)\left\langle n_{j}, n_{k}\right\rangle=0, \\
\left\langle n_{j}-n_{k}, n_{j}+n_{k}-H\right\rangle \cdot\left\langle n_{j}, n_{k}\right\rangle=0
\end{gathered}
$$

для любых значений индексов $j, k$. 
Условие (3.14) принимает следующий вид:

$$
\left(n_{j}-n_{k}\right) \cdot\left\langle n_{j}, n_{k}\right\rangle=0
$$

для любых $j, k$. Импликация $(3.18) \Rightarrow(3.16)$ (или $(3.18) \Rightarrow(3.17))$ очевидна.

Таким образом, каждое из условий (3.16) и (3.17) является условием полупараллельности тензора Риччи, а (3.18) - условием полупараллельности второй фундаментальной формы нормально плоского подмногообразия.

Лемма 3.2. Для того чтобъ нормально плоское подмногообразие $M$ в $E_{n}$ имело полупараллельный тензор Риччи, необходимо и достаточно, чтобы его главные векторы кривизны $n_{j}$ и вектор средней кривизны Н удовлетворяли условию (3.16) (или (3.17)).

Если $M$ является нормально плоским и минимальным, т.е. $H=0$, то из (3.16) следует, что $\left(\left|n_{j}\right|-\left|n_{k}\right|\right) \cdot\left\langle n_{j}, n_{k}\right\rangle=0$ для любых $j, k$. Итак, справедлива следующая

Лемма 3.3. Нормально плоское минимальное подмногообразие $M$ в $E_{n}$ имеет полупараллельный тензор Риччи тогда и только тогда, когда любые его два главных вектора кривизны либо равны по модулю, либо ортогоналъны.

Из этой леммы следует, что ненулевые главные векторы кривизны нормально плоского минимального подмногообразия с полупараллельным тензором Риччи разбиваются на группы, которые обладают следующими свойствами:

(а) любые два вектора, принадлежащие одной группе, имеют равные модули;

(б) любые два вектора, принадлежащие разным группам, взаимно ортогональны;

(в) сумма всех векторов, принадлежащих одной и той же группе, равна нулевому вектору.

Из свойства (в) следует, что каждая группа содержит не менее двух векторов.

Справедлива следующая

Лемма 3.4. Нормалъно плоское минималъное подмногообразие $M$ в $E_{n}$ является эйнштейновым тогда и только тогда, когда все его главные векторы кривизны имеют равные постоянные модули, т.е.

$$
\left|n_{1}\right|=\cdots=\left|n_{m}\right|=\rho=\text { const. }
$$

Эта лемма следует непосредственно из формулы (3.15), а также из того общеизвестного факта, что если $\operatorname{dim} M \geqslant 3$ и $R_{1}=\rho I$, то $\rho=$ const (см., например, [32]).

\section{§4. Схемы главных векторов кривизны нормально плоского минимального полуэйнштейнова подмногообразия}

Пусть $M$ является $m$-мерным нормально плоским полуэйнштейновым подмногообразием в $E_{n}$. Тогда тензор Риччи $R_{1}$ имеет на $T_{x}^{(0)}$ нулевое собственное значение, а на его ортогональном дополнении $T_{x}^{(1)}$ - только одно ненулевое собственное значение. Пусть $\operatorname{dim} T_{x}^{(0)}=\mu, \operatorname{dim} T_{x}^{(1)}=m-\mu$, и пусть адаптированный к $M$ ортонормрепер $\left\{x, e_{1}, \ldots, e_{m}, e_{m+1}, \ldots, e_{n}\right\}$ выбран так, что

$$
e_{a} \in T_{x}^{(1)}, \quad e_{r} \in T_{x}^{(0)}, \quad e_{\alpha} \in T_{x}^{\perp}(M),
$$


где $a=1, \ldots, m-\mu, r=m-\mu+1, \ldots, m, \alpha=m+1, \ldots, n$. Тогда $\rho_{a}=\rho$ $(\neq 0), \rho_{r}=0$, а из $(3.15)$ следует, что $\left|n_{r}\right|\left(\left|n_{r}\right|-|H| \cdot \cos \varphi_{r}\right)=0$, где $\varphi_{r}-$ угол между $n_{r}$ и $H$. Следовательно, либо $n_{r}=0$, либо $\left|n_{r}\right|=|H| \cdot \cos \varphi_{r}$. Если $n_{m-\mu+1}=0, \ldots, n_{m-\mu+\nu}=0, n_{m-\mu+\nu+1} \neq 0, \ldots, n_{m} \neq 0$, то индекс относительной дефектности подмногообразия $M$ равен $\nu$.

В дальнейшем будут рассматриваться только нормально плоские минимальные полуэйнштейновы подмногообразия, однако здесь мы отметим, что исследование нормально плоских полуэйнштейновых подмногообразий, удовлетворяющих условиям $\nu=0, \mu \geqslant 1$, представляет особый интерес, так как такие подмногообразия не содержат прямых (см. §2).

Итак, пусть $M$ является нормально плоским минимальным полуэйнштейновым подмногообразием в $E_{n}$ с индексом дефектности $\mu \geqslant 1$. Тогда из (3.15) при $H=0$ имеем $\rho_{a}=\left|n_{a}\right|^{2}=\rho(\neq 0), \rho_{r}=0$ и, следовательно, $n_{r}=0$. Таким образом, нормальные векторы $n_{1}, \ldots, n_{m-\mu}$ являются ненулевыми, имеют равные модули и их сумма равна нулевому вектору. Если откладывать векторы $n_{1}, \ldots, n_{m-\mu}$ от точки $x \in M$, то их концы будут принадлежать некоторой гиперсфере $S \subset T_{x}^{\perp}(M)$ (возможно, сфере меньшей размерности) радиуса $\left|n_{r}\right|=\sqrt{\rho}$ с центром в точке $x$. Условие минимальности подмногообразия $\left(n_{1}+\cdots+n_{m-\mu}=0\right)$ налагает определенные ограничения на взаимное расположение векторов $n_{1}, \ldots, n_{m-\mu}$. Каждое возможное расположение этих векторов будем называть схемой главных векторов кривизны. Например, если $M$ является гиперповерхностью, то возможна только схема 1.

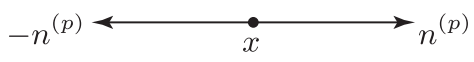

\section{Cxema 1}

На этой схеме запись $n^{(p)}$ означает, что главный вектор кривизны $n$ имеет кратность $p$. В этом случае имеется только два ненулевых главных вектора кривизны $n$ и $-n$ одинаковой кратности $p \geqslant 1$, образующих между собой угол в $180^{\circ}$. Если коразмерность подмногообразия $M$ равна двум, то возможна как схема 1, так и, например, схема 2.

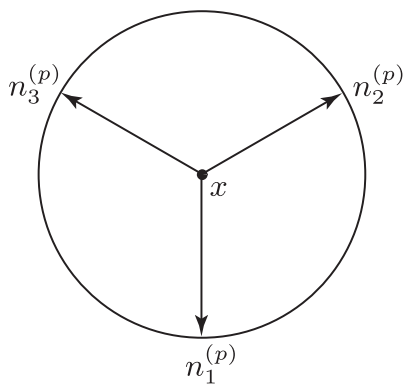

Cxema 2

В этом случае имеется только три ненулевых главных вектора кривизны одинаковой кратности $p$, образующие между собой углы в $120^{\circ}$. Однако такое 
расположение трех главных векторов кривизны, вообще говоря, не является единственно возможным.

Действительно, пусть коразмерность подмногообразия $\geqslant 2$, и пусть имеется только три ненулевых главных вектора кривизны $n_{1}, n_{2}, n_{3}$ с кратностями $p_{1}, p_{2}, p_{3}$ соответственно. Из условия $p_{1} n_{1}+p_{2} n_{2}+p_{3} n_{3}=0$ следует, что кратности $p_{1}, p_{2}, p_{3}$ "составляют" треугольник. Так как $\left|n_{1}\right|^{2}=\left|n_{2}\right|^{2}=\left|n_{3}\right|^{2}=\rho$, то, возводя равенства

$$
p_{1} n_{1}=-p_{2} n_{2}-p_{3} n_{3}, \quad p_{2} n_{2}=-p_{1} n_{1}-p_{3} n_{3}, \quad p_{3} n_{3}=-p_{1} n_{1}-p_{2} n_{2}
$$

в скалярный квадрат и сокращая на $\rho$, получаем

$$
\left\{\begin{array}{l}
p_{1}^{2}=p_{2}^{2}+2 p_{2} p_{3} \cos \varphi+p_{3}^{2} \\
p_{2}^{2}=p_{1}^{2}+2 p_{1} p_{3} \cos \psi+p_{3}^{2} \\
p_{3}^{2}=p_{1}^{2}+2 p_{1} p_{2} \cos \theta+p_{2}^{2}
\end{array}\right.
$$

где $\varphi$ - угол между $n_{2}$ и $n_{3}, \psi$ - угол между $n_{1}$ и $n_{3}, \theta$ - угол между $n_{1}$ и $n_{2}$. Так как $n_{2} \neq n_{3}, n_{2} \neq-n_{3}$, то $0<\varphi<\pi$. Точно так же $0<\psi<\pi, 0<\theta<\pi$, причем $\varphi+\psi+\theta=2 \pi$. Если известны кратности $p_{1}, p_{2}, p_{3}$, то по формулам (4.1) можем определить углы $\varphi, \psi, \theta$.

Отметим, что число треугольников с целочисленными сторонами и периметром $N$ дается определенной таблицей (см. [36; задача 284]).

Пусть $p_{1}=p_{2}=p_{3}$. Тогда из (4.1) следует, что $\cos \varphi=\cos \psi=\cos \theta=-1 / 2$, следовательно, $\varphi=\psi=\theta=120^{\circ}$. Обратно, если $\cos \varphi=\cos \psi=\cos \theta$, то, приравнивая значения этих косинусов из системы (4.1) и преобразуя, получаем

$\left(p_{1}-p_{2}\right)\left(p_{1}+p_{2}+p_{3}\right)\left(p_{1}+p_{2}-p_{3}\right)=0, \quad\left(p_{2}-p_{3}\right)\left(p_{1}+p_{2}+p_{3}\right)\left(p_{2}+p_{3}-p_{1}\right)=0$.

Отсюда следует, что $p_{1}=p_{2}=p_{3}$. Так как в наших рассуждениях коразмерность подмногообразия не играла существенной роли, то справедлива

ЛЕмма 4.1. Если нормально плоское минимальное полуэйнштейново подмногообразие $M$ в $E_{n}$ имеет только три различных ненулевых главных вектора кривизны $n_{1}, n_{2}, n_{3}$, то углы между ними равны тогда и только тогда, когда равны их кратности.

Покажем, что в этой лемме необходимость условий имеет общий характер. Действительно, пусть подмногообразие $M$ имеет главные векторы кривизны $n_{1}, \ldots, n_{q}$ с кратностями $p_{1}, \ldots, p_{q}$, и пусть $\varphi_{i j}$ - угол между $n_{i}$ и $n_{j}$. Умножая условие минимальности $p_{1} n_{1}+p_{2} n_{2}+\cdots+p_{q} n_{q}=0$ скалярно на $n_{1}, n_{2}, \ldots, n_{q}$ и сокращая на $\left|n_{1}\right|^{2}=\cdots=\left|n_{q}\right|^{2}=\rho$, получаем

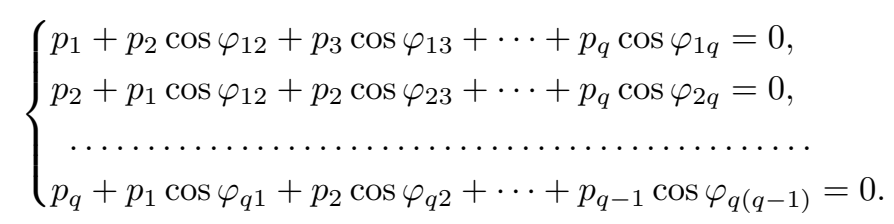


Пусть $\varphi_{i j}=\varphi$ для любых $i, j$. Тогда система (4.2) будет иметь следующий вид:

$$
\left\{\begin{array}{c}
p_{1}+\left(p_{2}+p_{3}+\cdots+p_{q}\right) \cos \varphi=0, \\
p_{2}+\left(p_{1}+p_{3}+\cdots+p_{q}\right) \cos \varphi=0, \\
\left.\ldots \ldots \cdots \cdots \cdots \cdots \cdots \cdots \cdots \cdots \cdots \cdots+p_{q-1}\right) \cos \varphi=0 \\
p_{q}+\left(p_{1}+p_{2}+\cdots+\right.
\end{array}\right.
$$

Исключая $\cos \varphi$ из первых двух уравнений системы $(4.3)$, получаем $\left(p_{2}-p_{1}\right) \times$ $\left(p_{1}+p_{2}+\cdots+p_{q}\right)=0$. Следовательно, $p_{1}=p_{2}$. Точно так же доказывается, что $p_{2}=p_{3}=\cdots=p_{q}$. Складывая все уравнения системы (4.3) и делая необходимые сокращения, находим

$$
\cos \varphi=-\frac{1}{q-1},
$$

где $q \geqslant 2$. Итак, справедлива

ЛЕмма 4.2. Если нормально плоское минимальное полуэйнштейново подмногообразие $M$ в $E_{n}$ имеет $q$ ненулевых главных векторов кривизны $n_{1}, \ldots, n_{q}, q \geqslant 2$, которые образуют между собой равные углы, то кратности этих векторов равны, а косинус угла ч между этими векторами определяется по формуле (4.4).

В лемме 4.1 достаточные условия не имеют общего характера, так как возможна, например, схема с векторами $n_{1}^{(p)},-n_{1}^{(p)}, n_{2}^{(p)},-n_{2}^{(p)}, n_{3}^{(p)},-n_{3}^{(p)}$, где углы между $n_{1}^{(p)}, n_{2}^{(p)}$ и $n_{3}^{(p)}$ могут быть не равны между собой.

Рассмотрим подробно такие схемы главных векторов кривизны, в которых углы между векторами равны между собой.

Если коразмерность подмногообразия $M$ равна 1 , то очевидно, что возможна только схема 1. Если коразмерность равна двум, т.е. $n-m=2$, то возможны только схемы 1 и 2. Схема 2 получается из схемы 1 следующим образом. Пусть в схеме 1 (и во всех рассматриваемых в дальнейшем схемах) векторы могут свободно поворачиваться вокруг точки $x$. В точке $x$ строим третий вектор, перпендикулярный вектору $n$ и имеющий такой же модуль. Далее вытягиваем непрерывно всю фигуру в направлении построенного вектора. Углы между векторами начинают непрерывно меняться, причем угол между $n$ и $-n$ уменьшается (начиная от $180^{\circ}$ ), а углы между $n,-n$ и третьим вектором увеличиваются (начиная от $90^{\circ}$ ). Как только указанные углы становятся равными, мы получаем схему 2. Если $n-m=3$, то к схемам 1 и 2 добавляется только схема 3.

Схема 3 получается из схемы 2 следующим образом. В точке $x$ строим перпендикулярный к плоскости схемы 2 вектор $n_{4}$ с таким же модулем, что и у остальных векторов. Далее, из конца вектора $n_{4}$ вытягиваем непрерывно всю фигуру в направлении этого вектора. Углы между векторами $n_{1}, n_{2}, n_{3}$ непрерывно уменьшаются, а углы между $n_{1}$ и $n_{4}, n_{2}$ и $n_{4}, n_{3}$ и $n_{4}$ увеличиваются. Достигнув значения $\arccos (-1 / 3)$, все эти углы становятся равными, и получается схема 3 . Если $n-m=4$, то к схемам 1-3 добавляется только одна схема, которая получается из схемы 3 построением в точке $x$ вектора $n_{5}$ перпендикулярно пространству $E_{3}$ и вытягиванием всей фигуры в направлении 


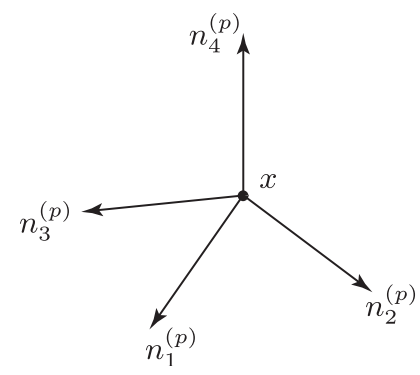

CXema 3

вектора $n_{5}$. Изменяясь непрерывно, углы между векторами достигают значения $\arccos (-1 / 4)$ и становятся равными. Получается очередная схема. На каждом шаге векторы направлены по образующим конуса соответствующей размерности с вершиной в точке $x$, а вновь построенный вектор направлен по оси конуса в сторону, противоположную направлению проекций предыдущих векторов на эту ось. По построению такая схема единственна с точностью до выбора вершины конуса и вращения схемы около вершины.

Продолжая описанную процедуру, получаем, что если коразмерность подмногообразия равна $n-m$, то в схемах главных векторов кривизны, в которых углы между всеми векторами попарно равны, максимальное число главных векторов кривизны может быть $n-m+1$.

В следующем параграфе мы даем геометрическое описание одного класса нормально плоских минимальных полуэйнштейновых подмногообразий со схемой главных векторов кривизны указанного вида.

\section{§ 5. Полуэйнштейновы подмногообразия с кратными главными векторами кривизны}

Пусть $M$ является $m$-мерным нормально плоским минимальным полуэйнштейновым подмногообразием индекса дефектности $\mu \geqslant 1$ в $n$-мерном евклидовом пространстве $E_{n}, n-m \geqslant 2$. Пусть в каждой точке $x \in M$ имеются $q \geqslant 3$ различных ненулевых главных векторов кривизны $n_{1}, \ldots, n_{q}$ кратностей $p_{1} \geqslant 2, \ldots, p_{q} \geqslant 2$ соответственно, $p_{1}+\cdots+p_{q}=m-\mu$. Как мы знаем,

$$
\begin{gathered}
p_{1} n_{1}+\cdots+p_{q} n_{q}=0, \\
\left|n_{1}\right|^{2}=\cdots=\left|n_{q}\right|^{2}=\rho .
\end{gathered}
$$

В дальнейшем будем считать, что обозначения $\S 3,4$ сохраняются, адаптированный к подмногообразию $M$ ортонормрепер $\left\{x, e_{1}, \ldots, e_{m}, e_{m+1}, \ldots, e_{n}\right\}$ выбран так же, как и в начале $\S 4$, а индексы принимают следующие значения:

$$
\begin{gathered}
i, j, k, \ldots=1, \ldots, m, \quad a, b, c, \ldots=1, \ldots, p_{1}+\cdots+p_{q}, \\
r, s, t, \ldots=p_{1}+\cdots+p_{q}+1, \ldots, m, \quad \varphi, \psi, \chi, \ldots=1, \ldots, q, \\
i_{\varphi}, j_{\varphi}, k_{\varphi}, \ldots=p_{1}+\cdots+p_{\varphi-1}+1, \ldots, p_{1}+\cdots+p_{\varphi-1}+p_{\varphi}, \\
\alpha, \beta, \gamma, \ldots=m+1, \ldots, n .
\end{gathered}
$$


Поскольку нормальная связность подмногообразия $M$ плоская, то в некотором адаптированном ортонормрепере все матрицы $\left\|h_{i j}^{\alpha}\right\|$ одновременно могут быть приведены к диагональному виду $\left\|\lambda_{i}^{\alpha} \delta_{i j}\right\|$. Тогда условие $n_{i_{\varphi}}=n_{j_{\varphi}}$ влечет $\lambda_{i_{\varphi}}^{\alpha}=\lambda_{j_{\varphi}}^{\alpha}=\lambda_{(\varphi)}^{\alpha}$. Следовательно, $n_{\varphi}=n_{i_{\varphi}}=n_{j_{\varphi}}=\lambda_{(\varphi)}^{\alpha} e_{\alpha}$ и условия (5.1), (5.2) принимают соответственно следующий вид:

$$
\sum_{\varphi} p_{\varphi} \lambda_{(\varphi)}^{\alpha}=0, \quad \sum_{\alpha}\left(\lambda_{(\varphi)}^{\alpha}\right)^{2}=\rho \quad \forall \varphi
$$

Из второго равенства в (5.3) получаем

$$
\sum_{\alpha} \lambda_{(\varphi)}^{\alpha} d \lambda_{(\varphi)}^{\alpha}=\frac{1}{2} d \rho .
$$

Каждая из матриц $\left\|h_{i j}^{\alpha}\right\|$ принимает следующий вид:

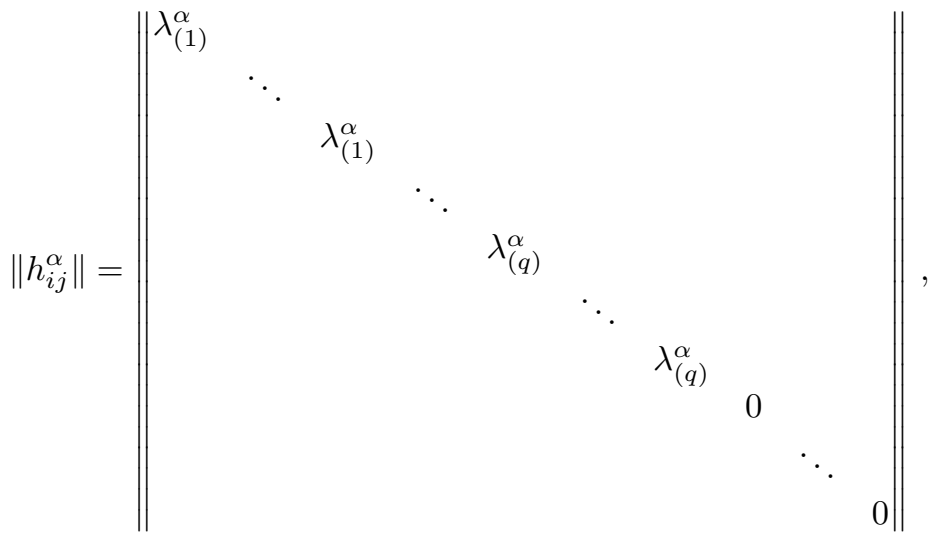

где $\lambda_{(\varphi)}^{\alpha}$ имеет кратность $p_{\varphi} \geqslant 2$ для любого значения $\varphi$, а $0-$ кратность $\mu \geqslant 1$.

Так как $n_{\varphi} \neq 0$, то $\lambda_{(\varphi)}^{\alpha} \neq 0$ при некотором значении индекса $\alpha$. Более того, так как $n_{\varphi} \neq n_{\psi}$ при $\varphi \neq \psi$, то $\lambda_{(\varphi)}^{\beta} \neq \lambda_{(\varphi)}^{\beta}$ при некотором значении индекса $\beta$.

Обратно, пусть в некотором поле адаптированного ортонормрепера к $m$-мерному подмногообразию $M$ евклидова пространства $E_{n}$ матрицы второй фундаментальной формы одновременно приводятся к виду (5.5), где $q \geqslant 2$, а диагональные элементы обладают вышеуказанными свойствами и выполняются условия (5.3). Тогда $M$ является нормально плоским минимальным полуэйнштейновым подмногообразием индекса дефектности $\mu \geqslant 1$ с $q$ различными ненулевыми главными векторами кривизны $n_{1}, \ldots, n_{q}$ кратностей $p_{1} \geqslant 2, \ldots$, $p_{q} \geqslant 2$ соответственно.

Пусть $T_{x}^{(0)}$ - пространство дефектности подмногообразия $M$ в точке $x$, и пусть $T_{x}^{(1, \varphi)}$ обозначает подпространство касательного пространства $T_{x}(M)$, на котором каждая матрица $\left\|h_{i j}^{\alpha}\right\|$ имеет собственное значение $\lambda_{(\varphi)}^{\alpha}$ кратности $p_{\varphi}$. Именно в этом смысле мы будем в дальнейшем говорить, что $T_{x}^{(1, \varphi)}$ является собственным подпространством, соответствующим главному вектору кривизны $n_{\varphi}$, а $T^{(1, \varphi)}$ - собственным распределением, соответствующим этому вектоpy. Тогда $\operatorname{dim} T_{x}^{(0)}=\mu, \operatorname{dim} T_{x}^{(1, \varphi)}=p_{\varphi}$ и справедливо разложение в прямую сумму:

$$
T_{x}(M)=T_{x}^{(1)}+T_{x}^{(0)}=T_{x}^{(1,1)}+\cdots+T_{x}^{(1, q)}+T_{x}^{(0)} .
$$


Основная цель настоящего параграфа - доказательство следующей теоремы.

ТЕОРема 5.1. Пусть в евклидовом пространстве $E_{n}$ т-мерное нормалъно плоское минимальное полуэйнштейново подмногообразие $M$ индекса дефектности $\mu \geqslant 1$ имеет в каждой точке $q$ ненулевых главных векторов кривизнъь $n_{1}, \ldots, n_{q}$ с кратностями $p_{1} \geqslant 2, \ldots, p_{q} \geqslant 2$ соответственно, $3 \leqslant q \leqslant$ $n-m+1$. Если соответствующие этим векторам собственные распределения $T^{(1,1)}, \ldots, T^{(1, q)}$ параллельны на $M$ друг относительно друга (но не относительно распределения $\left.T^{(0)}\right)$, то

(1) векторы $n_{1}, \ldots, n_{q}$ образуют попарно равные угль и $p_{1}=\cdots=p_{q}(=p)$,

(2) $M$ локально представляет собой прямое произведение $\widetilde{M} \times \widetilde{P}$, где $\widetilde{M}-$ $(\mu-1)$-мерная плоскость, а подмногообразие $\widetilde{P}$ несет $(q+1)$-компонентную ортогоналъную сопряженную систему, состоящую из q одинаковых сфер $S_{1}^{p}(R), \ldots, S_{q}^{p}(R), p \geqslant 2$, и прямой $L$, и представляет собой конус (с образующей $L$ в каждой точке) над прямым произведением $S_{1}^{p}(R) \times \cdots \times S_{q}^{p}(R)$, которое

(a) является $(p q+1)$-мерным эйнштейновым подмногообразием евклидова пространства $E_{n-\mu+1}$,

(б) принадлежит гиперсфере $S^{n-\mu}(\bar{R})$ пространства $E_{n-\mu+1}$ и является минимальным в этой гиперсфере; радиусы $R$ и $\bar{R}$ связаны условием $\bar{R}^{2}=q \cdot R^{2}$ и являются линейными (не постоянными) бункииями на $L$.

ДокАЗАТЕЛЬСтво. Будем предполагать, что ортонормрепер

$$
\left\{x, e_{1}, \ldots, e_{m}, e_{m+1}, \ldots, e_{n}\right\}
$$

выбран так, что в каждой точке $x \in M$ для каждого значения $\varphi$ векторы $e_{i_{\varphi}}$ принадлежат пространству $T_{x}^{(1, \varphi)}$, а векторы $e_{r}$ - пространству $T_{x}^{(0)}$. Через $T^{(1, \varphi)}, T^{(0)}$ будем обозначать соответствующие распределения.

Поскольку нормальная связность подмногообразия $M$ плоская, то нормальные к $M$ векторные поля $e_{\alpha}$ можно выбрать так, чтобы они были параллельными в нормальном расслоении, что равносильно $\omega_{\beta}^{\alpha}=0$ (см. [34; гл. 4, 1 , предложение 1.1]). В дальнейшем будем предполагать, что $e_{\alpha}$ так и выбраны.

Рассмотрим уравнения (3.1) и (3.2), которые в настоящем случае принимают следующий вид:

$$
\begin{gathered}
\omega^{\alpha}=0, \quad \omega_{i}^{\alpha}=\lambda_{i}^{\alpha} \delta_{i j} \omega^{j}, \\
d \lambda_{i}^{\alpha} \delta_{i j}+\left(\lambda_{j}^{\alpha}-\lambda_{i}^{\alpha}\right) \omega_{j}^{i}=h_{i j k}^{\alpha} \omega^{k},
\end{gathered}
$$

где в левой части уравнения (5.7) нет суммирования по $i$. С учетом принятых обозначений имеем $\lambda_{i_{\varphi}}^{\alpha}=\lambda_{(\varphi)}^{\alpha}, \lambda_{r}^{\alpha}=0$. Отсюда и из (5.6) следует, что $\omega_{r}^{\alpha}=0$.

Пусть в (5.7) $i=i_{\varphi}, j=j_{\varphi}, i_{\varphi} \neq j_{\varphi}$. Тогда левая часть равна нулю и, следовательно,

$$
h_{i_{\varphi} j_{\varphi} k}^{\alpha}=0, \quad i_{\varphi} \neq j_{\varphi} .
$$

Если в (5.7) $i=i_{\varphi}, j=j_{\psi}, \varphi \neq \psi$, то

$$
\left(\lambda_{(\psi)}^{\alpha}-\lambda_{(\varphi)}^{\alpha}\right) \omega_{j_{\psi}}^{i_{\varphi}}=h_{i_{\varphi} j_{\psi} k}^{\alpha} \omega^{k} .
$$


В силу условия теоремы распределения $T^{(1, \varphi)}$ и $T^{(1, \psi)}$ параллельны при $\varphi \neq \psi$. Это равносильно условию $\omega_{j_{\psi}}^{i_{\varphi}}=0, \varphi \neq \psi$, что в свою очередь влечет $h_{i_{\varphi} j_{\psi} k}^{\alpha}=0$. Таким образом,

$$
h_{i_{\varphi} j_{\psi} k}^{\alpha}=0, \quad \omega_{j_{\psi}}^{i_{\varphi}}=0, \quad \varphi \neq \psi .
$$

Пусть теперь в (5.7) $i=i_{\varphi}, j=i_{\varphi}$. Тогда с учетом предыдущих равенств будем иметь $d \lambda_{(\varphi)}^{\alpha}=h_{i_{\varphi} i_{\varphi} i_{\varphi}}^{\alpha} \omega^{i_{\varphi}}+h_{i_{\varphi} i_{\varphi} r}^{\alpha} \omega^{r}$, где в первом слагаемом правой части нет суммирования по $i_{\varphi}$. Если в $(5.7) i=i_{\varphi}, j=j_{\varphi}$, причем $i_{\varphi} \neq j_{\varphi}$, то $d \lambda_{(\varphi)}^{\alpha}=$ $h_{j_{\varphi} j_{\varphi} j_{\varphi}}^{\alpha} \omega^{j_{\varphi}}+h_{j_{\varphi} j_{\varphi} r}^{\alpha} \omega^{r}$, где в первом слагаемом правой части нет суммирования по $j_{\varphi}$. Сравнивая эти равенства и учитывая независимость форм $\omega^{i}$, получаем $h_{i_{\varphi} i_{\varphi} i_{\varphi}}^{\alpha}=0, h_{i_{\varphi} i_{\varphi} r}^{\alpha}=h_{j_{\varphi} j_{\varphi} r}^{\alpha}, i_{\varphi} \neq j_{\varphi}$. Таким образом,

$$
d \lambda_{(\varphi)}^{\alpha}=h_{i_{\varphi} i_{\varphi} r}^{\alpha} \omega^{r} .
$$

Умножая обе части этого равенства на $\lambda_{(\varphi)}^{\alpha}$ и суммируя по $\alpha$, будем иметь

$$
\sum_{\alpha} \lambda_{(\varphi)}^{\alpha} d \lambda_{(\varphi)}^{\alpha}=\sum_{\alpha} \lambda_{(\varphi)}^{\alpha} h_{i_{\varphi} i_{\varphi} r}^{\alpha} \omega^{r}
$$

Отсюда с учетом (5.4) следует, что

$$
\sum_{\alpha} \lambda_{(\varphi)}^{\alpha} h_{i_{\varphi} i_{\varphi} r}^{\alpha} \omega^{r}=\frac{1}{2} d \rho
$$

для любого $\varphi$ и, следовательно,

$$
\sum_{\alpha} \lambda_{(\varphi)}^{\alpha} h_{i_{\varphi} i_{\varphi} r}^{\alpha} \omega^{r}=\sum_{\alpha} \lambda_{(\psi)}^{\alpha} h_{i_{\psi} i_{\psi} r}^{\alpha} \omega^{r}
$$

для любых $\varphi, \psi, \varphi \neq \psi$.

Если в (5.7) $i=r, j=s$, то левая часть равна нулю и, следовательно, $h_{r s k}^{\alpha}=0$.

Если же в (5.7) $i=r, j=j_{\varphi}$, то с учетом предыдущих равенств получаем

$$
\lambda_{(\varphi)}^{\alpha} \omega_{j_{\varphi}}^{r}=h_{r j_{\varphi} j_{\varphi}}^{\alpha} \omega^{j_{\varphi}}
$$

где в правой части нет суммирования по $j_{\varphi}$. Умножая это равенство на $\lambda_{(\varphi)}^{\alpha}$ и суммируя по $\alpha$, будем иметь

$$
\left(\sum_{\alpha}\left(\lambda_{(\varphi)}^{\alpha}\right)^{2}\right) \omega_{j_{\varphi}}^{r}=\left(\sum_{\alpha} \lambda_{(\varphi)}^{\alpha} h_{r j_{\varphi} j_{\varphi}}^{\alpha}\right) \omega^{j_{\varphi}},
$$

где в правой части нет суммирования по $j_{\varphi}$. Отсюда, учитывая $(5.3)$ и $(5.12)$, получаем

$$
\omega_{j_{\varphi}}^{r}=F_{r} \omega^{j_{\varphi}},
$$

где $F_{r}$ равно отношению коэффициента правой части равенства (5.14) к коэффициенту левой части, который равен квадрату модуля вектора $n_{\varphi}$ и отличен от нуля. 
Если в $(5.13) \lambda_{(\varphi)}^{\alpha}=0$ при некотором значении индекса $\alpha$, то $h_{r j_{\varphi} j_{\varphi}}^{\alpha}=0$. Если же $\lambda_{(\varphi)}^{\alpha} \neq 0$ при некотором значении $\alpha$, то из (5.13) находим

$$
\omega_{j_{\varphi}}^{r}=\frac{h_{r j_{\varphi} j_{\varphi}}}{\lambda_{(\varphi)}^{\alpha}} \omega^{j_{\varphi}},
$$

где в правой части нет суммирования по $j_{\varphi}$. Сравнивая это равенство с $(5.15)$, получаем

$$
F_{r}=\frac{h_{r j_{\varphi} j_{\varphi}}^{\alpha}}{\lambda_{(\varphi)}^{\alpha}} .
$$

Используя это равенство, легко преобразовать уравнение (5.10) к следующему виду:

$$
d \ln \left|\lambda_{(\varphi)}^{\alpha}\right|=F_{r} \omega^{r} .
$$

Отсюда следует, что собственные значения $\lambda_{(\varphi)}^{\alpha}$ при всевозможных значениях индексов $\alpha$ и $\varphi$ различаются на постоянные множители. Поэтому в дальнейшем будем считать, что $\lambda_{(1)}^{m+1} \neq 0$, и рассматривать из системы (5.16) только первое уравнение (где $\alpha=m+1, \varphi=1)$.

Таким образом, имеем следующую систему:

$$
d \ln \left|\lambda_{(1)}^{m+1}\right|=F_{r} \omega^{r}, \quad \omega_{j_{\varphi}}^{r}=F_{r} \omega^{j_{\varphi}}, \quad \omega_{j_{\psi}}^{i_{\varphi}}=0, \quad \varphi \neq \psi
$$

Покажем, что распределения $T^{(1, \varphi)}$ и $T^{(0)}$ вполне интегрируемы, и выясним, что представляют собой их интегральные многообразия.

Распределение $T^{(1, \varphi)}$ задается следующей пфаффовой системой:

$$
\omega^{\alpha}=0, \quad \omega^{i_{\psi}}=0, \quad \psi \neq \varphi, \quad \omega^{r}=0 .
$$

Так как в этом случае (см. (5.17))

$$
\lambda_{(1)}^{m+1}=\mathrm{const}, \quad \omega_{j_{\varphi}}^{r}=F_{r} \omega^{j_{\varphi}}, \quad \omega_{j_{\psi}}^{r}=0, \quad \psi \neq \varphi, \quad \omega_{j_{\chi}}^{i \psi}=0,
$$

то, учитывая, что $\omega_{i_{\varphi}}^{\alpha}=\lambda_{(\varphi)}^{\alpha} \omega^{i \varphi}$, получаем

$$
d \omega^{\alpha}=0, \quad d \omega^{i} \psi=0, \quad \psi \neq \varphi, \quad d \omega^{r}=0
$$

Следовательно, система (5.18) вполне интегрируема, а из

$$
\omega_{i_{\varphi}}^{\alpha}=\lambda_{(\varphi)}^{\alpha} \omega^{i_{\varphi}}, \quad \omega_{j_{\varphi}}^{r}=F_{r} \omega^{j_{\varphi}}, \quad \omega_{i_{\varphi}}^{j_{\psi}}=0, \quad \psi \neq \varphi
$$

следует, что ее интегральное многообразие максимальной размерности является вполне омбилическим в $E_{n}$ (но не вполне геодезическим, так как $\lambda_{\varphi}^{\alpha} \neq 0$ при некотором значении индекса $\alpha$ ), т.е. локально является $p_{\varphi}$-мерной сферой, которую обозначим через $S^{p}\left(R_{\varphi}\right)$. Так как вектор средней кривизны $H_{\varphi}$ этой сферы определяется равенством

$$
H_{\varphi}=\lambda_{(\varphi)}^{\alpha} e_{\alpha}+\sum_{r} F_{r} e_{r}
$$


то для радиуса $R_{\varphi}$ получаем следующую формулу:

$$
R_{\varphi}^{2}=\frac{1}{\left|H_{\varphi}\right|^{2}}=\left(\sum_{\alpha}\left(\lambda_{(\varphi)}^{\alpha}\right)^{2}+\sum_{r} F_{r}^{2}\right)^{-1}
$$

Отсюда и из (5.3) следует, что все сферы имеют один и тот же радиус, т.е. $R_{1}=\cdots=R_{q}=R$.

Распределение $T^{(0)}$ задается пфаффовой системой $\omega^{\alpha}=0, \omega^{i}=0$, которая, как легко проверяется, вполне интегрируема. В силу соотношений $\omega_{r}^{\alpha}=0$, $\omega_{r}^{i_{\varphi}}=0$ (см. (5.17)) интегральное многообразие $M^{(0)}$ этой системы является вполне геодезическим в $E_{n}$, т.е. $\mu$-мерной плоскостью.

Пусть в $(5.17) \lambda_{(1)}^{m+1}=$ const (тогда все $\lambda_{(\varphi)}^{\alpha}$ постоянны). В этом случае

$$
F_{r}=0, \quad \omega_{j_{\varphi}}^{r}=0, \quad \omega_{j_{\psi}}^{i_{\varphi}}=0, \quad \varphi \neq \psi,
$$

и распределения $T^{(1, \varphi)}$ и $T^{(0)}$ параллельны на подмногообразии $M$. Так как они сопряжены относительно второй фундаментальной формы (все матрицы $\left\|h_{i j}^{\alpha}\right\|$ имеют диагональный вид), то $M$ локально является прямым произведением сфер $S^{p_{\varphi}}\left(R_{\varphi}\right)$ и плоскости $M^{(0)}$ (теорема 2.1). Такое произведение не может быть минимальным, поскольку вектор $n_{\varphi}$ является вектором средней кривизны (с учетом того, что $\left.F_{r}=0\right)$ сферы $S^{p_{\varphi}}\left(R_{\varphi}\right)$ и отличен от нуля по условию.

В дальнейшем будем считать, что $\lambda_{(1)}^{m+1}$ не является постоянной функцией.

Рассмотрим пфаффову систему

$$
\omega^{\alpha}=0, \quad \omega^{r}=0,
$$

которая задает распределение $K=T^{(1,1)}+\cdots+T^{(1, q)}$, сопоставляющее каждой точке $x \in M$ прямую сумму $K_{x}=T_{x}^{(1,1)}+\cdots+T_{x}^{(1, q)}$. В силу формул $(5.6)$ и (5.17) имеем

$$
d \omega^{\alpha}=\sum_{\varphi} \sum_{i_{\varphi}} \lambda_{(\varphi)}^{\alpha} \omega^{i_{\varphi}} \wedge \omega^{i_{\varphi}}=0, \quad d \omega^{r}=F_{r} \sum_{\varphi} \sum_{i_{\varphi}} \omega^{i_{\varphi}} \wedge \omega^{i_{\varphi}}=0 .
$$

Следовательно, рассматриваемая пфаффова система вполне интегрируема. Ее интегральное многообразие максимальной размерности будем обозначать через $\widetilde{K}$. Очевидно, что $\operatorname{dim} \widetilde{K}=p_{1}+\cdots+p_{q}$. Из формул (5.17) следует, что $\widetilde{K}$ является омбилическим относительно направлений $e_{r}$. Так как $\omega_{i_{\varphi}}^{\alpha}=\lambda_{(\varphi)}^{\alpha} \omega^{i_{\varphi}}$, то в направлениях $e_{\alpha}$ матрицы второй фундаментальной формы подмногообразия $\widetilde{K}$ также имеют диагональный вид. Следовательно, распределения $T^{(1,1)}, \ldots, T^{(1, q)}$ сопряжены относительно второй фундаментальной формы и в силу $\omega_{j_{\psi}}^{i_{\varphi}}=0, \varphi \neq \psi$, они параллельны на $\widetilde{K}$. Тогда $\widetilde{K}$ локально является прямым произведением сфер $S^{p_{1}}\left(R_{1}\right), \ldots, S^{p_{q}}\left(R_{q}\right)$.

Так как все матрицы второй фундаментальной формы подмногообразия $\widetilde{K}$ одновременно приведены к диагональному виду, то его нормальная связность является плоской. Из условия $\omega_{r}^{\alpha}=0$ следует, что $T^{(0)}$ как подрасслоение нормального расслоения для $\widetilde{K}$ является параллельным и, следовательно, в нем индуцируется плоская связность. Это значит, что нормальные к $\widetilde{K}$ векторные поля $e_{r}$ в $T^{(0)}$ можно выбрать так, что они будут параллельными в нормальном 
расслоении. При таком выборе векторных полей $e_{r}$ будем иметь $\omega_{r}^{S}=0$ (при $\left.\omega^{r}=0\right)$. Но поскольку в общем случае $\omega_{r}^{S}=\Gamma_{r k}^{s} \omega^{k}$, то $\Gamma_{r i_{\varphi}}^{s}=0$ для любого значения $\varphi$ и, следовательно, $\omega_{r}^{S}=\Gamma_{r t}^{s} \omega^{t}$.

Дифференцируя внешним образом уравнения системы (5.17) и применяя лемму Картана, получаем

$$
\begin{gathered}
d F_{r}-F_{s} \omega_{r}^{s}=P_{r s} \omega^{s}, \quad P_{r s}=P_{s r}, \\
d F_{r}-F_{s} \omega_{r}^{s}-F_{r} F_{s} \omega^{s}=Q_{r} \omega^{j_{\varphi}}, \\
\sum_{r}\left(F_{r}\right)^{2}+\sum_{\alpha} \lambda_{(\varphi)}^{\alpha} \lambda_{(\psi)}^{\alpha}=0 .
\end{gathered}
$$

Из второго уравнения в силу независимости форм $\omega^{j_{\varphi}}$ имеем $Q_{r}=0$. Тогда $P_{r s}=F_{r} F_{s}$ и, учитывая формулу, полученную для форм $\omega_{r}^{s}$, преобразуем последнюю систему к следующему виду:

$$
\begin{gathered}
d F_{r}=\left(F_{s} \Gamma_{r t}^{s}+F_{r} F_{t}\right) \omega^{t}, \\
\sum_{\alpha} \lambda_{(\varphi)}^{\alpha} \lambda_{(\psi)}^{\alpha}=-\sum_{r}\left(F_{r}\right)^{2}, \quad \varphi \neq \psi .
\end{gathered}
$$

Здесь второе уравнение имеет следующий геометрический смысл: если подмногообразие $\widetilde{K}$ мы рассматриваем с точки зрения объемлющего пространства $E_{n}$, то секционные кривизны между сферами $S^{p_{1}}\left(R_{1}\right), \ldots, S^{p_{q}}\left(R_{q}\right)$ равны нулю. Если же $\widetilde{K}$ мы рассматриваем как подмногообразие в $M$, то на $M$ секционные кривизны между этими сферами равны между собой и все отрицательны. Это значит, что скалярные произведения главных векторов кривизны $n_{1}, \ldots, n_{q}$ отрицательны и равны между собой. Так как модули этих векторов равны, то равны и отрицательны косинусы углов между этими векторами. Следовательно, все эти углы равны некоторому углу $\varphi, 90^{\circ}<\varphi \leqslant 180^{\circ}$. На основании леммы 4.2 заключаем, что $p_{1}=p_{2}=\cdots=p_{q}=p$, a $\cos \varphi$ определяется по формуле (4.4). Таким образом, кратности всех ненулевых главных векторов кривизны равны между собой. Следовательно, сферы $S^{p_{1}}\left(R_{1}\right), \ldots, S^{p_{q}}\left(R_{q}\right)$ имеют одинаковые размерности и радиусы.

Поскольку

$$
\sum_{\alpha} \lambda_{(\varphi)}^{\alpha} \lambda_{(\psi)}^{\alpha}=\left\langle n_{\varphi}, n_{\psi}\right\rangle=\rho \cos \varphi=-\frac{\rho}{q-1},
$$

то систему (5.19) можем преобразовать к следующему виду:

$$
\begin{gathered}
d F_{r}=\left(F_{s} \Gamma_{r t}^{s}+F_{r} F_{t}\right) \omega^{i} \\
\sum_{r}\left(F_{r}\right)^{2}=\frac{\rho}{q-1} .
\end{gathered}
$$

Продолжим изучение подмногообразия $\widetilde{K}$. Из $(5.17)$ и $(5.20)$ следует, что $\lambda_{(1)}^{m+1}$ и $F_{r}$ постоянны на $\widetilde{K}$. Так как на $\widetilde{K}$ имеют место равенства $\omega_{r}^{\alpha}=0, \omega_{r}^{S}=0$, то нормальное к $\widetilde{K}$ векторное поле

$$
\xi=\sum_{r} F_{r} e_{r}
$$


параллельно в нормальном расслоении подмногообразия $\widetilde{K}$. Выбирая параллельное нормальное к $\widetilde{K}$ векторное поле $e_{m}$ так, что $\xi=\eta e_{m}, \eta>0$, получаем $F_{r}=0, r=q \cdot p+1, \ldots, m-1, F_{m}=\eta$. Отождествляя точку $x \in \widetilde{K}$ с радиусвектором, будем иметь

$$
d\left(x+\eta^{-1} e_{m}\right)=\omega^{a} e_{a}+\eta^{-1} \omega_{m}^{a} e_{a}=\omega^{a} e_{a}+\eta^{-1}\left(-\eta \omega^{a} e_{a}\right)=0 .
$$

Следовательно, $x+\eta^{-1} e_{m}=$ const и подмногообразие $\widetilde{K}$ принадлежит некоторой гиперсфере $S^{n-1}(\bar{R})$ пространства $E_{n}$, для которой вектор $e_{m}$ является нормальным. Поскольку $\bar{R}^{2}=1 / \eta^{2}, R_{1}^{2}=\cdots=R_{q}^{2}=R^{2}=\left(\rho+\eta^{2}\right)^{-1}$, причем $\rho=(q-1) \eta^{2}$ (см. второе равенство в $\left.(5.20)\right)$, то $R_{1}^{2}+\cdots+R_{q}^{2}=\bar{R}^{2}$ или $\bar{R}^{2}=q R^{2}$.

Так как подмногообразие $M$ является минимальным и $F_{r}=0$ при $r \neq m$, то нормальный к $\widetilde{K}$ вектор $\eta e_{m}$ является вектором средней кривизны для $\widetilde{K}$. Однако поскольку вектор $e_{m}$ ортогонален к гиперсфере $S^{n-1}(\bar{R})$, то $\widetilde{K}$ является минимальным в этой гиперсфере, но не минимальным в $E_{n}$. Подмногообразие $\widetilde{K}$, являясь прямым произведением $q$ одинаковых сфер, т.е. $q$ эйнштейновых подмногообразий с одинаковыми эйнштейновыми константами, также является эйнштейновым с такой же эйнштейновой константой (см., например, [26; гл. 1 , предложение 1.99]).

Продолжим изучение систем (5.17) и (5.20). Учитывая, что $F_{m}=\eta>0$, $F_{r}=0$ при $r \neq m$, из (5.20) получим $\Gamma_{r t}^{m}=0$. Это позволяет преобразовать системы (5.17) и (5.20) к следующему виду:

$$
\begin{gathered}
d \ln \left|\lambda_{(1)}^{m+1}\right|=\eta \omega^{m}, \quad \omega_{j_{\varphi}}^{m}=\eta \omega^{j_{\varphi}}, \\
\omega_{j_{\varphi}}^{r}=0, \quad r \neq m, \quad \omega_{j_{\psi}}^{i_{\varphi}}=0, \quad \varphi \neq \psi, \\
d \eta=\eta^{2} \omega^{m}, \quad \eta^{2}=\frac{\rho}{q-1} .
\end{gathered}
$$

Поскольку $\Gamma_{r t}^{m}=0$, то $\omega_{r}^{m}=0$ и распределение $\bar{T}$, сопоставляющее каждой точке подмногообразия $M$ линейную оболочку векторов $e_{p q+1}, \ldots, e_{m-1}$, является параллельным на $M$ и, следовательно, вполне интегрируемым. Интегральное многообразие $\widetilde{M}$ этого распределения является $(\mu-1)$-мерной плоскостью. Ортогональное дополнение $P_{x}$ к $\bar{T}_{x}$ в касательном пространстве $T_{x}(M)$ имеет вид $T_{x}^{(1,1)}+\cdots+T_{x}^{(1, q)}+L_{x}$, где $L_{x}-$ прямая с направляющим вектором $e_{m}$. Соответствующее распределение $P$ также параллельно и вполне интегрируемо. В силу сопряженности распределений $\bar{T}$ и $P=T^{(1,1)}+\cdots+T^{(1, q)}+L$ относительно второй фундаментальной формы подмногообразия $M$ заключаем, что $M$ локально является прямым произведением их интегральных многообразий $\widetilde{M}$ и $\widetilde{P}$ соответственно. Здесь $\widetilde{P}$ является $(p q+1)$-мерным полуэйнштейновым подмногообразием в $(n-\mu+1)$-мерном евклидовом пространстве $E_{n-\mu+1}$. Подмногообразие $\widetilde{P}$ несет $(q+1)$-компонентную сопряженную систему, состоящую из $q$ одинаковых сфер и прямой. Прямое произведение этих сфер является эйнштейновым подмногообразием в $E_{n-\mu+1}$ и принадлежит гиперсфере этого пространства.

Поскольку $\widetilde{M}$ является евклидовым пространством размерности $\mu-1$, то в качестве локальных координат на $\widetilde{M}$ можно взять прямоугольные декартовы координаты. Тогда $\omega_{s}^{r}=0$ для любых $r, s=p q+1, \ldots, m-1$. Следовательно, $\Gamma_{s t}^{r}=0$ для любых $r, s, t=p q+1, \ldots, m$. 
На подмногообразии $\widetilde{P}$ интегральное многообразие распределения $L$ есть прямая с единичным вектором $e_{m}$. Обозначим через $x^{m}$ координату на этой прямой. Так как $d \omega^{m}=0$ (что легко проверяется), то можем считать, что $\omega^{m}=d x^{m}$. Подставляя в $(5.21)$ и интегрируя, находим

$$
\eta=\frac{1}{C-x^{m}}, \quad \rho=\frac{q-1}{\left(C-x^{m}\right)^{2}}, \quad \lambda_{(1)}^{m+1}=\frac{C_{1}^{m+1}}{C-x^{m}},
$$

где $C$ и $C_{1}^{m+1}$ - постоянные интегрирования, причем $C_{1}^{m+1} \neq 0$. Так как в $(5.21)$ в первом уравнении индекс $m+1$ можно заменить произвольным значением индекса $\alpha$, то все ненулевые $\lambda_{(\varphi)}^{\alpha}$ определяются по такой же формуле, т.е.

$$
\lambda_{(\varphi)}^{\alpha}=\frac{C_{\varphi}^{\alpha}}{C-x^{m}} .
$$

Из последнего уравнения системы (5.21) в силу того, что

$$
\rho=\left|n_{\varphi}\right|^{2}=\sum_{\alpha}\left(\lambda_{(\varphi)}^{\alpha}\right)^{2}=\frac{1}{\left(C-x^{m}\right)^{2}} \sum_{\alpha}\left(C_{\varphi}^{\alpha}\right)^{2},
$$

следует, что $C_{\varphi}^{\alpha}$ должны удовлетворять условию

$$
\sum_{\alpha}\left(C_{\varphi}^{\alpha}\right)^{2}=q-1
$$

для любого значения индекса $\varphi$. Кроме того, $C_{\varphi}^{\alpha}$ должны удовлетворять также условию

$$
\sum_{\varphi} C_{\varphi}^{\alpha}=0
$$

которое получается из первого равенства системы (5.3) после подстановки значений $\lambda_{(\varphi)}^{\alpha}$ и учета того, что $p_{\varphi}=p$. Далее, так как

$$
\sum_{\alpha} \lambda_{(\varphi)}^{\alpha} \lambda_{(\psi)}^{\alpha}=-\frac{\rho}{q-1}
$$

то, подставляя сюда значения $\lambda_{(\varphi)}^{\alpha}, \lambda_{(\psi)}^{\alpha}$ и $\rho$, получаем, что $C_{\varphi}^{\alpha}$ должны удовлетворять также условию

$$
\sum_{\alpha} C_{\varphi}^{\alpha} C_{\psi}^{\alpha}=-1, \quad \varphi \neq \psi
$$

Покажем, что постоянные $C_{\varphi}^{\alpha}$, удовлетворяющие условиям (5.22)-(5.24), существуют. Действительно, пусть $N_{1}=\left(C_{1}^{\alpha}\right), \ldots, N_{q}=\left(C_{q}^{\alpha}\right)$ - система векторов $(q-1)$-мерного евклидова пространства, координаты которых определены в некотором ортонормированном базисе. Предположим, что все векторы исходят из одной точки, имеют одинаковые модули, равные $\sqrt{q-1}$, и попарно образуют между собой угол $\varphi$ такой, что $\cos \varphi=-1 /(q-1)$ (см. $\S 4)$. Тогда $\left|N_{\varphi}\right|^{2}=q-1$ (что равносильно (5.22)), $N_{1}+\cdots+N_{q}=0$ (что равносильно (5.23)), $\left\langle N_{\varphi}, N_{\psi}\right\rangle=\left|N_{\varphi}\right| \cdot\left|N_{\psi}\right| \cdot \cos \varphi=-1$ (что равносильно (5.24)). Таким образом, координаты векторов $N_{1}, \ldots, N_{q}$ удовлетворяют условиям (5.22)-(5.24). 
Теперь покажем, что подмногообразие $\widetilde{P}$ является конусом с одномерной плоской образующей над эйнштейновым подмногообразием $\widetilde{K}$. Действительно, так как

$$
d e_{m}=-\eta \sum_{\varphi} \omega^{i} e_{i_{\varphi}}
$$

то $e_{m}=$ const при $\omega^{i \varphi}=0$. Отсюда следует, что $\widetilde{P}$ является либо конусом (с исключенной точечной вершиной, ибо в ней теряется регулярность), либо цилиндром над $\widetilde{K}$ с одномерной образующей $L$. Поскольку $\eta \neq 0$, то $\widetilde{P}$ может быть только конусом.

Если $\lambda_{(\varphi)}^{\alpha}=0$ при некотором $\alpha$ и любом значении индекса $\varphi$, то $d e_{\alpha}=0$. Тогда подмногообразие $M$ содержится в некотором евклидовом пространстве $E_{n-1}$. Следовательно, в общем случае $M$ содержится в некотором евклидовом пространстве $E_{n^{\prime}}$, где $m+1 \leqslant n^{\prime} \leqslant n$. Если для любого значения $\alpha$ существует такое значение $\varphi$, что $\lambda_{(\varphi)}^{\alpha} \neq 0$, то $M$ содержится в $E_{n}$ и не содержится в ни в каком его собственном подпространстве.

Для завершения доказательства теоремы предположим, что $\eta, \rho$ и $\lambda_{(\varphi)}^{\alpha}$ определены как и выше и выполняются условия (5.22)-(5.24). Тогда

$$
\sum_{\varphi} \lambda_{(\varphi)}^{\alpha}=0, \quad \sum_{\alpha}\left(\lambda_{(\varphi)}^{\alpha}\right)^{2}=\eta^{2}(q-1), \quad \sum_{\alpha} \lambda_{(\varphi)}^{\alpha} \lambda_{(\psi)}^{\alpha}=-\eta^{2} .
$$

Рассмотрим в $E_{n}$ следующую дифференциальную систему:

$$
\begin{gathered}
\omega^{\alpha}=0, \quad \omega_{\beta}^{\alpha}=0, \quad \omega_{r}^{\alpha}=0, \quad \omega_{s}^{r}=0, \quad \omega_{j_{\psi}}^{i_{\varphi}}=0, \quad \varphi \neq \psi, \\
\omega_{i_{\varphi}}^{\alpha}-\lambda_{(\varphi)}^{\alpha} \omega^{i_{\varphi}}=0, \quad d \ln \left|\lambda_{(\varphi)}^{\alpha}\right|-\eta \omega^{m}=0, \quad d \eta-\eta^{2} \omega^{m}=0, \\
\omega_{j_{\varphi}}^{m}-\eta \omega^{j_{\varphi}}=0, \quad \omega_{j_{\varphi}}^{r}=0, \quad r \neq m .
\end{gathered}
$$

Путем непосредственного вычисления внешних дифференциалов левых частей уравнений этой системы легко убедиться в том, что все они обращаются в нуль в силу уравнений самой системы. Это значит, что данная система вполне интегрируема. Она фактически и задает описанное выше нормально плоское минимальное полуэйнштейново подмногообразие $M=\widetilde{M} \times \widetilde{P}$. Теорема 5.1 полностью доказана.

Отметим, что случай $q=2$ теоремы 5.1 фактически рассмотрен автором в [12] и [14]. В этом случае параллельность распределений $T^{(1, \varphi)}$ получается как следствие.

\section{Список литературы}

[1] S. Tanno, "Hypersurfaces satisfying a certain condition on the Ricci tensor", Tôhoku Math. J. (2), 21:2 (1969), 297-303.

[2] K. Sekigawa, "On some hypersurfaces satisfying $R(X, Y) \cdot R_{1}=0$ ", Hokkaido Math. J., $\mathbf{1 : 1}$ (1972), 102-109.

[3] K. Sekigawa, H. Takagi, "On conformally flat spaces satisfying a certain condition on the Ricci tensor", Tôhoku Math. J. (2), 23:1 (1971), 1-11.

[4] H. Nakagawa, R. Takagi, "Kaehler submanifolds with $R S=0$ in a complex projective space", Hokkaido Math. J., 5:1 (1976), 67-70. 
[5] Y. Matsuyama, "Complete hypersurfaces with $R S=0$ in $E^{n+1}$ ", Proc. Amer. Math. Soc., 88 (1983), 119-123.

[6] В. А. Мирзоян, "Ric-полусимметрические подмногообразия", Итоги науки и техники. Проблемы геометрии, 23, ВИНИТИ, М., 1991, 29-66.

[7] В. А. Мирзоян, "Подмногообразия с полупараллельным тензором Риччи", Ученые записки Тартуск. ун-та, 1991, №930, 113-128.

[8] В. А. Мирзоян, "Структурные теоремы для римановых Ric-полусимметрических пространств", Изв. вузов. Сер. матем., 1992, № 6, 80-89.

[9] В. А. Мирзоян, "Обобщения теоремы Ю. Лумисте о полупараллельных подмногообразиях", Изв. НАН Армении. Сер. матем., 33:1 (1998), 53-64.

[10] В. А. Мирзоян, "Конусы над эйнштейновыми пространствами”, Изв. НАН Армении. Сер. матем., 33:5 (1998), 46-54.

[11] В.А. Мирзоян, "Подмногообразия с параллельными и полупараллельными структурами", Изв. НАН Армении. Сер. матем., 34:5 (1999), 63-66.

[12] В. А. Мирзоян, "Классификация Ric-полупараллельных гиперповерхностей в евклидовых пространствах", Матем. сб., 191:9 (2000), 65-80.

[13] В. А. Мирзоян, "Подмногообразия с полупараллельными тензорными полями как огибающие", Матем. сб., 193:10 (2002), 99-112.

[14] В.А. Мирзоян, "Скрещенные произведения, конусы над эйнштейновыми пространствами и классификация одного класса Ric-полупараллельных подмногообразий", Изв. РАН. Сер. матем., 67:5 (2003), 107-124.

[15] F. Defever, "Ricci-semisymmetric hypersurfaces", Balkan J. Geom. Appl., 5:1 (2000), $81-91$.

[16] Ü. Lumiste, "Semiparallelity, semisymmetricity and Ric- semisymmetricity for normally flat submanifolds in Euclidean space", Proc. Estonian Acad. Sci. Phys. Math., $\mathbf{5 1 : 2}$ (2002), 67-85.

[17] D. Ferus, "Symmetric submanifolds of Euclidean space", Math. Ann., 247:1 (1980), 81-93.

[18] F. Dillen, S. Nölker, "Semi-parallelity, multi-rotation surfaces and the helix-property", J. Reine Angew. Math., 435 (1993), 33-63.

[19] Ü. Lumiste, "Submanifolds with parallel fundamental form", Handbook of differential geometry, vol. 1, eds. F. Dillen, L. Verstraelen, North-Holland, Amsterdam, 2000, 779-864.

[20] В.А. Мирзоян, "s-полупараллельные подмногообразия в пространствах постоянной кривизны как огибающие $s$-параллельных подмногообразий”, Изв. $H A H$ Армении. Матем., 31:5 (1996), 44-56.

[21] R. Deszcz, L. Verstraelen, S. Yaprak, "Pseudosymmetric hypersurfaces in 4-dimensional spaces of constant curvature", Bull. Inst. Math. Acad. Sinica, 22:2 (1994), $167-179$.

[22] F. Defever, R. Deszcz, L. Verstraelen, "On pseudosymmetric para-Kähler manifolds", Colloq. Math., 74:2 (1997), 253-260.

[23] K. Arslan, Y. Celik, R. Deszcz, R. Ezentaș, "On the equivalence of Ricci-semisymmetry and semisymmetry", Colloq. Math., 76:2 (1998), 279-294.

[24] Z. I. Szabó, "Structure theorems on Riemannian spaces satisfying $R(X, Y) \cdot R=0$. I. The local version", J. Differential Geom., 17:4 (1982), 531-582.

[25] А. З. Петров, Пространства Эйнштейна, Физматгиз, М., 1961.

[26] А. Бессе, Многообразия Эйнштейна, т. 1, 2, Мир, М., 1990.

[27] S. S. Chern, N. Kuiper, "Some theorems on the isometric imbedding of compact Riemannian manifolds in Euclidean space", Ann. of Math. (2), 56:3 (1952), 422-430.

[28] E. Boeckx, O. Kowalski, L. Vanhecke, Riemannian manifolds of conullity two, World Sci., Singapore, 1996. 
[29] П. А. Широков, "Постоянные поля векторов и тензоров 2-го порядка в римановых пространствах", Изв. физ.-матем. об-ва при Казанском ун-те. Сер. 2, 25 (1925), 86-114.

[30] В. А. Мирзоян, "Подмногообразие с параллельным тензором Риччи в евклидовых пространствах", Изв. вузов. Сер. матем., 1993, № 9, 22-27.

[31] Б. А. Дубровин, С. П. Новиков, А. Т. Фоменко, Современная геометрия. Методъ и приложения, Наука, Физматлит, М., 1986.

[32] М. М. Постников, Лекиии по геометрии. Семестр VI. Риманова геометрия, Факториал, М., 1998.

[33] Ш. Кобаяси, К. Номидзу, Основы дифференциальной геометрии, т. 2, Наука, Физматлит, М., 1981.

[34] B.-Y. Chen, Geometry of submanifolds, Pure Math. Appl., 22, Marcel Dekker, New York, 1973.

[35] J. D. Moore, "Isometric immersions of Riemannian products", J. Differential Geom., 5 (1971), 159-168.

[36] Н. Я. Виленкин, Комбинаторика, Наука, Физматлит, М., 1969.

В. А. Мирзоян (V. A. Mirzoyan)

Государственный инженерный университет Армении, Европейская региональная академия на Кавказе, г. Ереван E-mail: mirzoyan_vanya@front.ru
Поступила в редакцию 23.05.2005 